\title{
WIND TURBINE COLLECTIVE AND INDIVIDUAL PITCH \\ CONTROL USING QUANTITATIVE FEEDBACK THEORY
}

by

\section{LAURA WHEELER}

\author{
Submitted in partial fulfillment of the requirements for the degree of \\ Master of Science
}

Thesis Advisor: Dr. Mario Garcia-Sanz

Department of Electrical Engineering and Computer Science

CASE WESTERN RESERVE UNIVERSITY

May, 2017 


\title{
CASE WESTERN RESERVE UNIVERSITY SCHOOL OF GRADUATE STUDIES
}

\author{
We hereby approve the thesis of \\ Laura Wheeler \\ candidate for the degree of Master of Science*. \\ Committee Chair \\ Dr. Mario Garcia-Sanz \\ Committee Member \\ Dr. Kenneth Loparo \\ Committee Member \\ Dr. Sree N. Sreenath \\ Committee Member \\ Dr. Roberto Fernández Galán \\ Date of Defense
}

March 22, 2017

*We also certify that written approval has been obtained for any proprietary material contained therein. 


\section{Table of Contents}

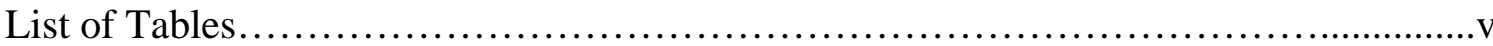

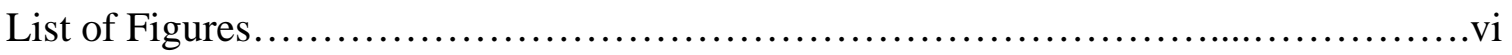

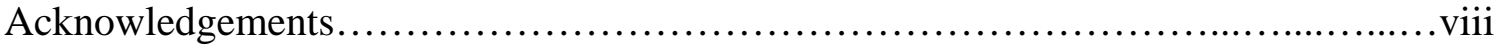

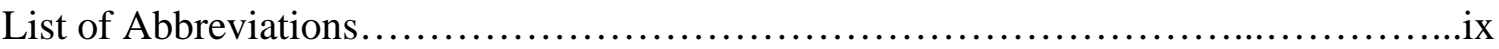

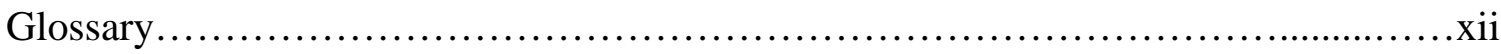

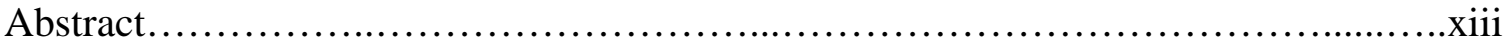

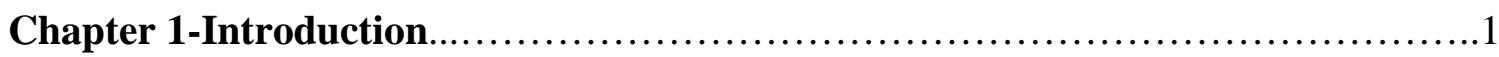

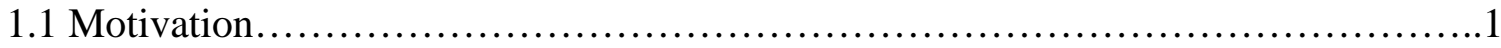

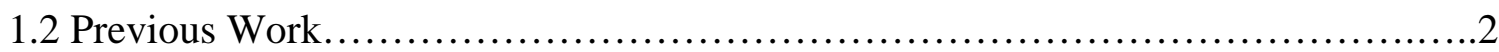

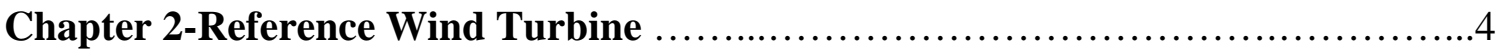

Chapter 3-Quantitative Feedback Theory .......................................

Chapter 4-Collective Pitch Control...............................................

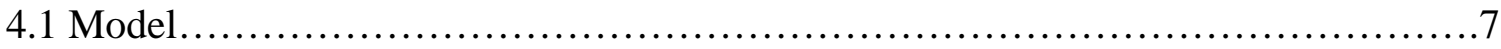

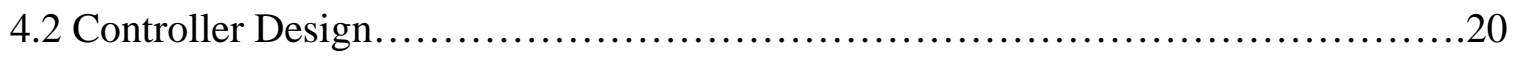

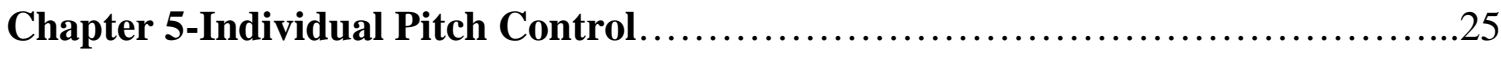

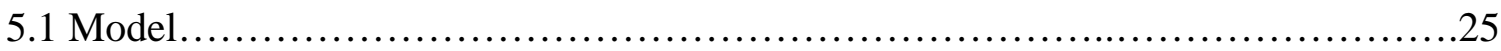

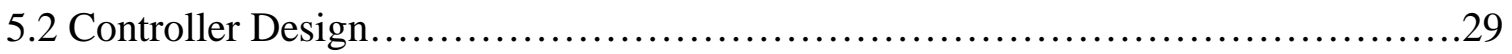

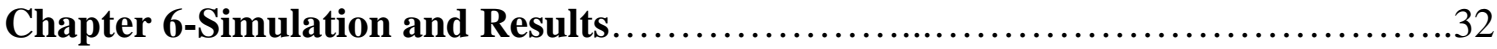

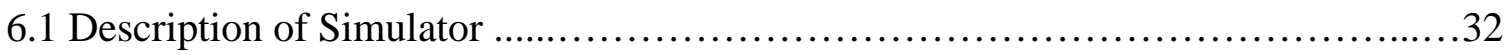

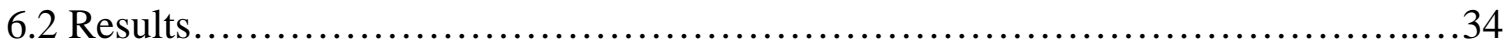

Chapter 7-Conclusions and Future Work .....................................47

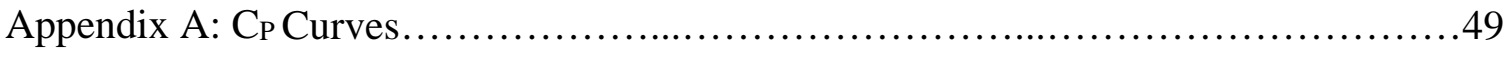


Appendix B: Bode Plots of CPC Nominal Plants......................................50

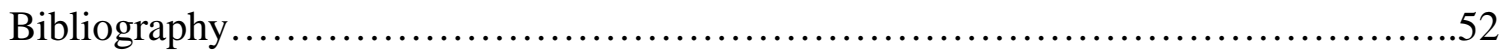




\section{List of Tables}

4.1 Working Points for Collective Pitch Control Model..............................18

4.2 Wind Turbine Parameters for Collective Pitch Control Model......................19

6.1 Bending Moment Reduction at Key Frequencies...............................41

6.2 Root Flapwise Bending Moment Reduction at Key Frequencies...................45

6.3 Bending Moment Reduction at Key Frequencies using Energy Measurement.........46

6.4 Root Flapwise Bending Moment Reduction at Key Frequencies using Energy

Measurement.................................................................... 46 


\section{List of Figures}

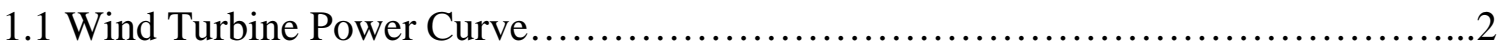

4.1 Detailed Block Diagram of the Collective Pitch Control Systems...................17

4.2 Reduced Block Diagram of the Collective Pitch Control Systems....................17

4.3 Simulink Model Used to Find Working Points..................................18

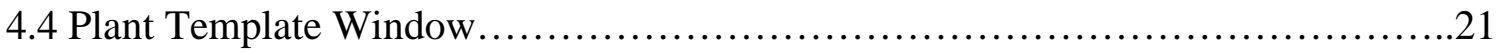

4.5 Intersection Bounds Window........................................................

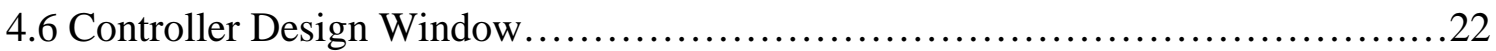

4.7 Stability Analysis Window......................................................

4.8 Disturbance Rejection Analysis Window............................................23

4.9 Step Response (Secondary Analysis Window) ................................24

5.1 Step Simulation to Find Individual Pitch Control Model............................28

5.2 Pitch Control Block for Step Simulation........................................28

6.1 Simulation for Collective Pitch Control Only ..................................32

6.2 Block Diagram of Collective Pitch Controller....................................33

6.3 Simulation for Collective and Individual Pitch Control............................33

6.4 Block Diagram of Collective and Individual Pitch Control..........................33

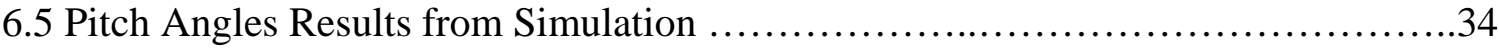

6.6 Sample of Pitch Angles Results from Simulation.....................................35

6.7 Generator Power Simulation Results.............................................35

6.8 Generator Torque Simulation Results.............................................

6.9 Rotor Speed Simulation Results..................................................

$6.10 \mathrm{M}_{\text {tilt }}$ Simulation Results.......................................................... 


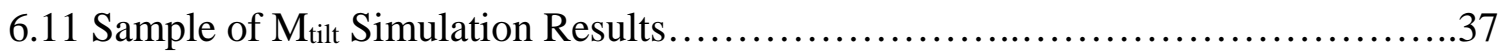

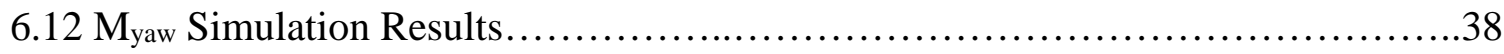

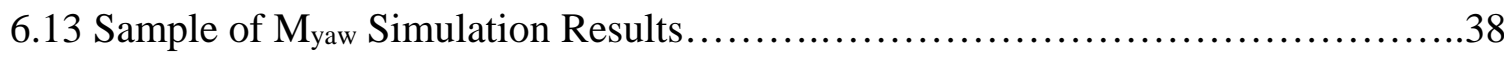

6.14 Magnitude of Fast Fourier Transform of $\mathrm{M}_{\text {tilt }}$ with a window of $200 \ldots \ldots \ldots \ldots \ldots . . .39$

6.15 Magnitude of Fast Fourier Transform of $\mathrm{M}_{\mathrm{tilt}}$ with a window of $30 \ldots \ldots \ldots \ldots \ldots \ldots . . . . .39$

6.16 Magnitude of Fast Fourier Transform of $M_{\text {yaw }}$ with a window of $200 \ldots \ldots \ldots \ldots \ldots . . .40$

6.17 Magnitude of Fast Fourier Transform of $\mathrm{M}_{\text {yaw }}$ with a window of $30 \ldots \ldots \ldots \ldots \ldots \ldots . .40$

6.18 Magnitude of Fast Fourier Transform of Bending Moment of Blade 1 with a window

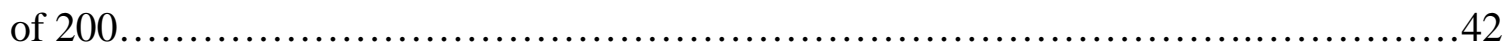

6.19 Magnitude of Fast Fourier Transform of Bending Moment of Blade 1 with a window

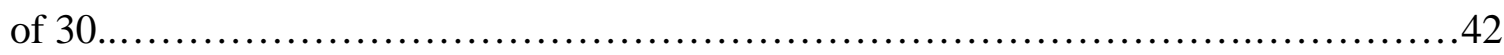

6.20 Magnitude of Fast Fourier Transform of Bending Moment of Blade 2 with a window

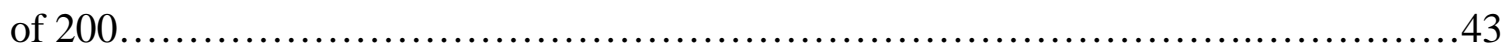

6.21 Magnitude of Fast Fourier Transform of Bending Moment of Blade 2 with a window

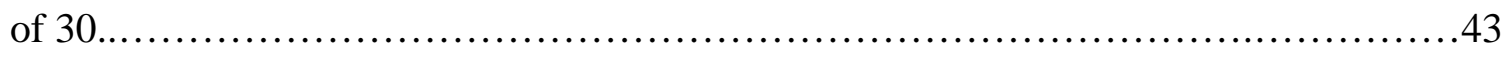
6.22 Magnitude of Fast Fourier Transform of Bending Moment of Blade 3 with a window

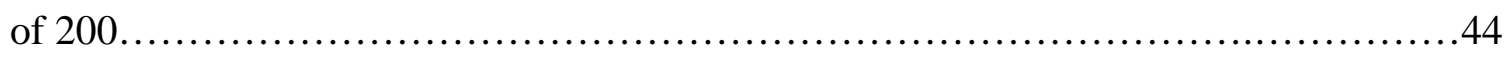

6.23 Magnitude of Fast Fourier Transform of Bending Moment of Blade 3 with a window

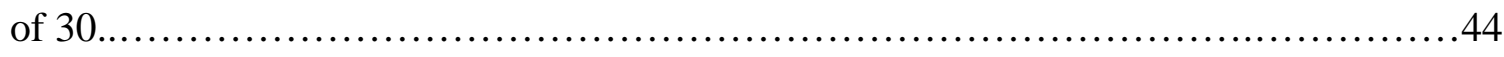

A.1 CP/ $\lambda$ Curve for the NREL 5 MW Reference Turbine...............................49

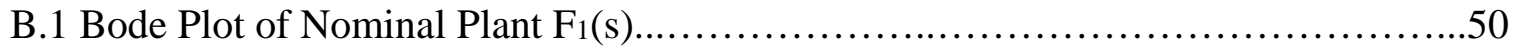

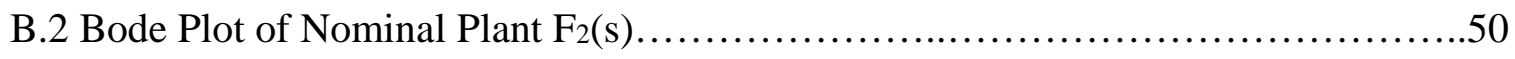

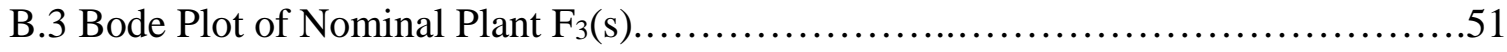




\section{Acknowledgements}

Completing this thesis would not have been possible with the guidance of my advisor, Dr. Mario Garcia-Sanz. I am also indebted to Fa Wang and Sameer Alsharif for their advice and assistance. I will always be grateful to my teammates on the CWRU Cross Country and Track Teams for their support and encouragement. Finally, I want to thank my parents, Mark Wheeler and Denise Hartsough, for their support. 


\section{List of Abbreviations}

\section{General}

CAE - Common Application Environment

CPC - Collective Pitch Control

FAST - Fatigue, Aerodynamics, Structures, and Turbulence

IPC - Individual Pitch Control

MIMO - Multi-Input Multi-Output

NREL - National Renewable Energy Laboratory

$P I$ - Proportional Integral

QFT - Quantitative Feedback Theory

QFTCT - Quantitative Feedback Theory Control Toolbox

SISO - Single Input Single Output

\section{Collective Pitch Control}

$a-$ axial induction factor

$B_{b}-$ damping coefficient of the rotor

$B_{s}$ - damping coefficient of the shaft

$B_{t}-$ damping coefficient of the tower

$C_{P}$ - power coefficient

$C_{T}-$ thrust coefficient

$D_{n}$ - dissipation function

$E_{k}-$ kinetic energy

$E_{P}-$ potential energy

$F_{T}-$ thrust force applied by the wind on the rotor 
$I_{g}$ - moment of inertia of the generator

$I_{r}$ - moment of inertia of the rotor

$K_{b}$ - spring constant of the rotor

$K_{s}$ - spring constant of the shaft

$K_{t}$ - spring constant of the tower

$K_{F \Omega}, K_{F V}, K_{F \beta}, K_{T \Omega}, K_{T V}, K_{T \beta}-$ gain elements of $\mathrm{F}_{\mathrm{T}}$ and $\mathrm{T}_{\mathrm{r}}$ transfer matrix

$m_{1}$ - mass of the tower

$m_{2}$ - mass of the nacelle, hub and blades

$N$ - number of blades

$q$ - generalized coordinate

$r_{b}$ - radius of the rotor

$T_{r}-$ aerodynamic torque applied by the wind on the rotor

$T_{g}$ - antagonic electrical torque applied on the shaft

$T_{g d}$ - demanded electrical torque

$v_{1}$ - upstream air velocity

$v_{2}$ - downstream air velocity

$y_{t}$-axial displacement of the nacelle

$\beta$ - pitch angle

$\beta_{d}$ - demanded pitch angle

$\gamma$ - angular displacement of the blades out of the plane of rotation

$\theta_{g}$ - generator angle position

$\theta_{r}$ - rotor angle position

$\lambda$ - tip speed ratio 
$\rho$ - air density

$\Omega_{r}$ - rotational speed/rotor speed

\section{Individual Pitch Control}

$f_{h}$ - high-corner frequency of the band pass filter

$f_{l}$ - low-corner frequency of the band pass filter

$\tilde{M}_{i}$ - perturbed blade root flap-wise bending moment for each blade i=1,2,3

$\mathrm{M}(t)$ - the averaged flap-wise bending moment

$M_{i}(t)$ - total blade root flap-wise bending moments for blades 1 , 2, and 3

$M_{\text {tilt,yaw }}$ - total blade root flap-wise bending moment in the tilt or yaw coordinate frame

$M_{x, i}$ - the effective flap-wise bending moment of each blade i=1,2,3

$\tilde{\theta}_{i}-$ perturbed blade pitch angle for each blade $\mathrm{i}=1,2,3$

$\theta(t)$ - averaged blade-pitch angle demand

$\theta_{i}$ - blade pitch angle for $\mathrm{i}=1,2,3$

$\theta_{\text {tilt,yaw }}$ total pitch angle in the tilt or yaw coordinate frame

$\phi(t)$ - rotor azimuth angle

$\Omega_{r}$ - rotor speed 


\section{Glossary}

- Nichols chart: Chart used in stability analysis where the phase of the system is plotted on the $\mathrm{x}$-axis and the gain of the system in $\mathrm{dB}$ is plotted on the $\mathrm{y}$-axis. Circles on the plot define all points where the closed-loop gain is constant [11].

- Rise time: Time for output $\mathrm{y}(\mathrm{t})$ to go from $10 \%$ of steady state value to $90 \%$ of steady state value [7].

- Settling time: Time from $\mathrm{t}=0$ seconds to time at which output $\mathrm{y}(\mathrm{t})$ remains within $2 \%$ of the steady state value [7]. 


\title{
Wind Turbine Collective and Individual Pitch Control Using Quantitative Feedback \\ Theory
}

\author{
Abstract \\ by
}

\section{LAURA WHEELER}

Individual pitch control is a new technique in the field of wind turbine control, used to reduce the asymmetric mechanical loads on the blades of multi-megawatt turbines. Therefore, the mechanical fatigue is reduced and the lifetime of the turbine is extended. In this work, an individual pitch controller is developed for the National Renewable Energy Laboratory’s (NREL) 5 MW reference wind turbine. The individual pitch controller works along with a collective pitch controller, designed using the Quantitative Feedback Theory Control Toolbox in Matlab.

The individual and collective pitch controllers are simulated using NREL's computer-aided engineering tool for horizontal axis wind turbines, FAST. Simulations show that the addition of the individual pitch controller reduces the loads on the tilt and yaw turbine components (the nacelle and tower) at the 1p and 3p frequencies by half, and the loads on the blades at the 2 p harmonic frequency, by almost half. 


\section{Chapter 1 - Introduction}

\subsection{Motivation}

Wind energy continues to grow throughout the world. In 2015, more new capacity was added in the wind energy sector than in any other power generation sector [10]. This is due to the creation of more wind farms and the development of larger capacity turbines. In North America, the average size of wind turbines installed was more than 2 MW in 2015, compared to 1.4 MW in 2005 [25]. The largest turbines in the world are now in the range of 5MW for commercial turbines, and in the range of $7 \mathrm{MW}$ for prototype turbines [1].

As wind turbines increase in size, the loads on the different components of the turbine increase, which can reduce turbine lifetime $[17,28]$. This is, in part, due to the unbalanced and changing loads on the wind turbine rotor caused by wind shear, gravitational force, and atmospheric turbulence [17]. However, most control methods assume that the wind is constant and uniform across the entire rotor. Collective pitch control (CPC), a commonly used method for regulating turbine speed, is based on this assumption [17].

The objective of CPC is to regulate rotor speed in Region 3 of the power curve pictured in Figure 1 below. In this region, when the turbine is operating at its rated power, the goal is to maintain power and rotor speed while rejecting wind disturbances. While the torque is held constant, the pitch is changed uniformly following the demands of a closed-loop rotor speed controller that limits energy capture and follows wind-speed variations [29]. 
To address the unbalanced and changing loads, research is now being done in the area of individual pitch control (IPC). In IPC, each individual blade is adjusted separately. The IPC system works with the CPC to address the asymmetries the CPC does not take into account. This alleviates structural and fatigue loads, which reduces turbine cost. The turbine then requires less maintenance, has a longer lifetime, and is more reliable [27, 28].

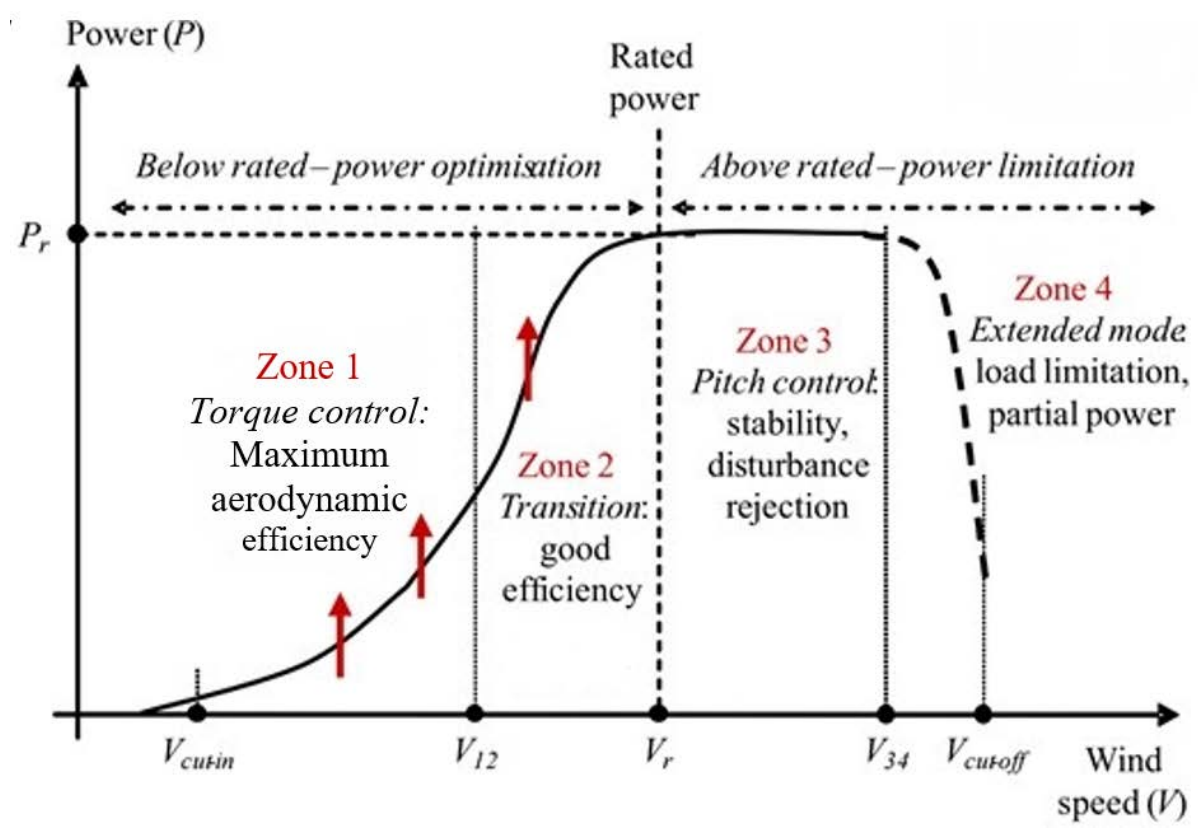

Figure 1.1: Wind Turbine Power Curve [6]

\subsection{Previous Work}

Over the past decade, researchers have used different approaches to design individual pitch controllers. Most of the proposed individual pitch controllers are based on optimal time-domain approaches such as linear quadratic Gaussian (LQG), linear quadratic regulators (LQR), multiblade coordinate (MBC), l1-optimal methods, and disturbance accommodating control [20, 21, 23, 24, 28]. However, the goal of individual pitch control is to reduce loads at specific frequencies, typically the $1 p$ and $3 p$ frequencies. It is 
difficult to look at performance at certain frequencies when working in the time domain. Therefore, frequency domain control techniques are better suited to IPC than time domain control techniques.

Both Lu et al. and Vali et al. have looked at IPC from the frequency domain perspective. Lu et al. use $\mathrm{H}_{\infty}$ loop shaping to design an individual pitch controller, while Vali et al. formulate a mixed sensitivity $\mathrm{H}_{\infty}$ optimization problem to design an optimal multivariable individual pitch controller. But there are several disadvantages to the $\mathrm{H}_{\infty}$ control technique. This technique is mathematically complex, and difficult to implement in real-world situations [19]. Additionally, when using $\mathrm{H}_{\infty}$ methods, the controller is typically very high order, which is not appropriate for practical implementation.

The remainder of the thesis is organized as follows. Chapter 2 discusses the reference wind turbine used in simulation. Chapter 3 provides an overview of Quantitative Feedback Theory, which is used for collective pitch control design. This is followed by chapters on the model and controller design for the collective pitch controller and individual pitch controller, respectively. The simulation and its results are covered in Chapter 6. Chapter 7 discusses the conclusions and possible future work. 


\section{Chapter 2 - Reference Wind Turbine}

The reference wind turbine used in this thesis is the 5MW Reference Turbine for Offshore System Development created by the National Renewable Research Laboratory (NREL) in Golden, CO. The reference turbine is not based on any one real turbine, instead it is a reasonable approximation of a multi-megawatt turbine. It is based on multiple real-world turbines and conceptual models of similar sizes [12].

The $5 \mathrm{MW}$ turbine is a horizontal axis wind turbine with three blades. Both variable speed generator torque control and variable individual pitch control can be used to control the 5MW Reference Turbine [12]. Although the reference turbine has been designed for use in simulations of offshore applications, the model can easily be used in onshore applications as well.

The reference turbine can be simulated in FAST, NREL's primary Common Application Environment. FAST, short for Fatigue, Aerodynamics, Structure and Turbulence, simulates the dynamic response of wind turbines. It joins together models of the relevant aerodynamics, servo dynamics, and structural dynamics to carry out nonlinear wind turbine simulation in the time domain [13].

The 5 MW turbine is used as a reference turbine in this study because information on the turbine is readily available. Many of the needed parameters can be obtained from technical reports from NREL. Also, simulations of the $5 \mathrm{MW}$ turbine can be carried out easily in FAST. Lastly, it makes sense to use a multi-megawatt turbine as the model in this study, because the size of wind turbines installed around the world continues to grow. 


\section{Chapter 3 - Quantitative Feedback Theory}

Quantitative Feedback Theory (QFT) is a control design method that uses feedback to reduce the effects of uncertainty in the plant and meet performance specifications [8]. The plant and the performance specifications are specified in the frequency domain. The plant is represented by a set of linear time invariant transfer functions over the range of any parametric uncertainty. The performance specifications are represented by two linear time invariant transfer functions that form the upper and lower boundaries [8].

To begin controller design, points within the range of each parameter's uncertainty, for each value of frequency $\omega=\omega_{\mathrm{i}}$, are plotted on the Nichols chart. For each $\omega_{\mathrm{i}}$, a contour (known as a template) is drawn through the data points [8]. This represents the region of structured plant parametric uncertainty on the Nichols chart, or the region where the controlled system meets the performance specifications [8].

QFT is well suited to design collective pitch controllers for several reasons. First of all, design specifications for collective pitch controllers are typically specified in the frequency domain [8], which fits with how specifications are represented in QFT. Additionally, because the models used to develop collective pitch controllers ignore some of the dynamics of an actual turbine, robust control techniques are preferred as they result in controllers not sensitive to unmodelled dynamics. This can be represented as uncertainty in QFT.

The collective pitch controller in this thesis is designed using the Quantitative Feedback Theory Control Toolbox (QFTCT) developed by Garcia-Sanz. It is an interactive object-oriented Matlab toolbox for QFT robust control systems design [2]. QFTCT has been used in both industry and academic projects [2]. The user is able to 
enter the system plant (with or without uncertainties) and specifications into the toolbox.

Then the user is able to add and modify controllers, adjusting the controllers based on the built-in QFT principles. 


\section{Chapter 4 - Collective Pitch Control}

\subsection{Model}

The plant used to design the collective pitch controller is a four-mass model of a variable speed direct drive (gearless) wind turbine. Although the NREL 5 MW turbine has a gearbox, values are provided for the equivalent shaft, not the high and low speed shafts separately [12]. Therefore, the best model for a collective pitch controller for the NREL 5 MW turbine is a direct drive model. The model used in this thesis is based on the model derived by Garcia-Sanz and Houpis in Wind Energy Systems.

Garcia-Sanz and Houpis identify the parameters of the four-mass model as follows: the wind applies an aerodynamic torque $T_{r}$ on the rotor. On the other end of the system, the electronic converter applies an antagonic electrical torque $\mathrm{T}_{\mathrm{g}}$ on the shaft. The rotor has a moment of inertia $\mathrm{I}_{\mathrm{r}}$, and the generator has a moment of inertia $\mathrm{Ig}_{\mathrm{g}}$. The shaft has a torsional stiffness coefficient $\mathrm{K}_{\mathrm{s}}$, and a viscous damping coefficient $\mathrm{B}_{\mathrm{s}}$ [8]. The excitation current, $I_{x}$, is the current introduced into the rotor. Then the active and reactive powers (P and $\mathrm{Q}$ ) are supplied to the grid. At the grid connection point, the frequency, voltage, and power factor of the grid are represented by $f, U$, and $\varphi$ [8].

There are four degrees of freedom in the system: rotor angle $\left(\Theta_{\mathrm{r}}\right)$, generator angle $\left(\Theta_{\mathrm{g}}\right)$, axial displacement of the nacelle $\left(\mathrm{y}_{\mathrm{t}}\right)$, and the angular displacement of the blades (pitch angle, or $\beta$ ). Therefore, there are four generalized coordinates of the system, $q$. The generalized coordinates of the system, q:

$$
q=\left[q_{i}\right]=\left[\begin{array}{llll}
y_{t} & \gamma & \theta_{r} & \theta_{g}
\end{array}\right]^{T}
$$


The kinetic energy of the system:

$$
E_{k}=\frac{m_{1}}{2} y_{t}^{2}+\frac{m_{2}}{2}\left(r_{b} \gamma^{\prime}+y_{t}^{\prime}\right)^{2}+\frac{I_{r}}{2} \theta_{r}^{\prime 2}+\frac{I_{g}}{2} \theta_{g}^{\prime 2}
$$

The potential energy of the system:

$$
E_{p}=\frac{K_{t}}{2} y_{t}^{2}+\frac{N K_{b}}{2}\left(r_{b} \gamma\right)^{2}+\frac{K_{s}}{2}\left(\theta_{r}-\theta_{g}\right)^{2}
$$

The dissipation function:

$$
D_{n}=\frac{B_{t}}{2} y_{t}^{\prime 2}+\frac{N B_{b}}{2}\left(r_{b} \gamma^{\prime}\right)^{2}+\frac{B_{s}}{2}\left(\theta_{r}^{\prime}-\theta_{g}^{\prime}\right)^{2}
$$

The Euler-Lagrange equation:

$\frac{d}{d t}\left(\frac{\partial L}{\partial q_{i}^{\prime}}\right)-\frac{\partial L}{\partial q_{i}}+\frac{\partial D_{N}}{\partial q_{i}}=Q_{i} \quad \mathrm{i}=1,2, \ldots$ number of degrees of freedom

where $\mathrm{L}$ is the Lagrangian function $\mathrm{L}=\mathrm{E}_{\mathrm{k}}-\mathrm{E}_{\mathrm{p}}$ [8].

Then Garcia-Sanz and Houpis derive the Lagrange equation terms from equations (1)-(4), where $\frac{\partial E_{p}}{\partial q_{i}}=0$ and $\frac{\partial E_{k}}{\partial q_{i}}=0$.

$$
\frac{d}{d t}\left(\frac{\partial L}{\partial q_{i}^{\prime}}\right)=\frac{d}{d t}\left[\begin{array}{c}
\frac{\partial E_{k}}{\partial y_{t}^{\prime}} \\
\frac{\partial E_{k}}{\partial \gamma^{\prime}} \\
\frac{\partial E_{k}}{\partial \theta_{r}^{\prime}} \\
\frac{\partial E_{k}}{\partial \theta_{g}^{\prime}}
\end{array}\right]=\frac{d}{d t}\left[\begin{array}{c}
\left(m_{1}+m_{2}\right) y_{t}^{\prime}+m_{2} r_{b} \gamma^{\prime} \\
m_{2} r_{b}^{2} \gamma^{\prime}+m_{2} r_{b} y_{t}^{\prime} \\
I_{r} \theta_{r}^{\prime} \\
I_{g} \theta_{g}^{\prime}
\end{array}\right]=\left[\begin{array}{cccc}
m_{1}+m_{2} & m_{2} r_{b} & 0 & 0 \\
m_{2} r_{b} & m_{2} r_{b}^{2} & 0 & 0 \\
0 & 0 & I_{r} & 0 \\
0 & 0 & 0 & I_{g}
\end{array}\right]\left[\begin{array}{l}
y_{t}^{\prime \prime} \\
\gamma^{\prime \prime} \\
\theta_{r}^{\prime \prime} \\
\theta_{g}^{\prime \prime}
\end{array}\right]=M q^{\prime \prime}
$$




$$
\begin{aligned}
& -\frac{\partial L}{\partial q_{i}}=\frac{\partial E_{p}}{\partial q_{i}}=\left[\begin{array}{c}
\frac{\partial E_{p}}{\partial y_{t}} \\
\frac{\partial E_{p}}{\partial \gamma} \\
\frac{\partial E_{p}}{\partial \theta_{r}} \\
\frac{\partial E_{p}}{\partial \theta_{g}}
\end{array}\right]=\left[\begin{array}{c}
K_{t} y_{t} \\
N K_{b} r_{b}^{2} \gamma \\
K_{s} \theta_{r}-K_{s} \theta_{g} \\
K_{s} \theta_{g}-K_{s} \theta_{r}
\end{array}\right]=\left[\begin{array}{cccc}
K_{t} & 0 & 0 & 0 \\
0 & N K_{b} r_{b}^{2} & 0 & 0 \\
0 & 0 & K_{s} & -K_{s} \\
0 & 0 & -K_{s} & K_{s}
\end{array}\right]\left[\begin{array}{c}
y_{t} \\
\gamma \\
\theta_{r} \\
\theta_{g}
\end{array}\right]=K q \\
& \frac{\partial D_{n}}{\partial q_{i}^{\prime}}=\left[\begin{array}{c}
\frac{\partial D_{n}}{\partial y_{t}^{\prime}} \\
\frac{\partial D_{n}}{\partial \gamma^{\prime}} \\
\frac{\partial D_{n}}{\partial \theta_{r}^{\prime}} \\
\frac{\partial D_{n}}{\partial \theta_{g}^{\prime}}
\end{array}\right]=\left[\begin{array}{c}
B_{t} y_{t}^{\prime} \\
N B_{b} r_{b}^{2} \gamma^{\prime} \\
B_{s} \theta_{r}^{\prime}-B_{s} \theta_{g}^{\prime} \\
B_{s} \theta_{g}^{\prime}-B_{s} \theta_{r}^{\prime}
\end{array}\right]=\left[\begin{array}{cccc}
B_{t} & 0 & 0 & 0 \\
0 & N B_{b} r_{b}^{2} & 0 & 0 \\
0 & 0 & B_{s} & -B_{s} \\
0 & 0 & -B_{s} & B_{s}
\end{array}\right]\left[\begin{array}{c}
y_{t}^{\prime} \\
\gamma^{\prime} \\
\theta_{r}^{\prime} \\
\theta_{g}^{\prime}
\end{array}\right]=C q^{\prime} \\
& Q=\left[\begin{array}{c}
F_{T} \\
r_{p} F_{T} \\
T_{r} \\
-T_{g}
\end{array}\right]=\left[\begin{array}{ccc}
1 & 0 & 0 \\
r_{p} & 0 & 0 \\
0 & 1 & 0 \\
0 & 0 & -1
\end{array}\right]\left[\begin{array}{c}
F_{T} \\
T_{r} \\
T_{g}
\end{array}\right]=R u
\end{aligned}
$$

Using equations 6-9, equation 5 becomes $M q^{\prime \prime}+C q^{\prime}+K q=Q\left(q^{\prime}, q, u, t\right)$. Rearranging the previous equation, equation 5 becomes

$$
q^{\prime \prime}=-M^{-1} C q^{\prime}-M^{-1} K q+M^{-1} R u
$$

To describe the system in State Space form, Garcia-Sanz and Houpis define three vectors:

State variables: $x=\left[\begin{array}{llllllll}y_{t} & \gamma & \theta_{r} & \theta_{g} & y_{t}^{\prime} & \gamma^{\prime} & \theta_{r}^{\prime} & \theta_{g}^{\prime}\end{array}\right]^{T}$

Inputs: $u=\left[\begin{array}{lll}F_{T} & T_{r} & T_{g}\end{array}\right]^{T}$

Outputs: $y=\left[\begin{array}{cccc}y_{t}^{\prime} & \gamma^{\prime} & \theta_{r}^{\prime} & \theta_{g}^{\prime}\end{array}\right]^{T}$

Following the standard State Space description $\left(\boldsymbol{x}^{\prime}=\boldsymbol{A x}+\boldsymbol{B y}, \boldsymbol{y}=\mathbf{C x}\right)$, they find: 


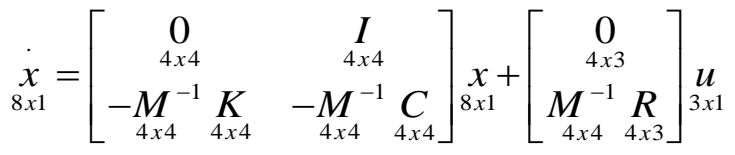

$$
\begin{aligned}
& \underset{4 \times 1}{y}=\left[\begin{array}{cc}
0 & I \\
4 \times 4 & I_{4 \times 4}
\end{array}\right]_{8 \times 1}^{x}
\end{aligned}
$$

The matrix $\boldsymbol{M}^{-1}$ :

$$
M^{-1}=\left[\begin{array}{cccc}
\frac{1}{m_{1}} & \frac{-1}{m_{1} r_{b}} & 0 & 0 \\
\frac{-1}{m_{1} r_{b}} & \frac{m_{1}+m_{2}}{m_{1} m_{2} r_{b}^{2}} & 0 & 0 \\
0 & 0 & \frac{1}{I_{r}} & 0 \\
0 & 0 & 0 & \frac{1}{I_{g}}
\end{array}\right]
$$

The matrices of the State Space description:

$$
A=\left[\begin{array}{cccccccc}
0 & 0 & 0 & 0 & 1 & 0 & 0 & 0 \\
0 & 0 & 0 & 0 & 0 & 1 & 0 & 0 \\
0 & 0 & 0 & 0 & 0 & 0 & 1 & 0 \\
0 & 0 & 0 & 0 & 0 & 0 & 0 & 1 \\
\frac{-K_{t}}{m_{1}} & \frac{N K_{b} r_{b}}{m_{1}} & 0 & 0 & \frac{-B_{t}}{m_{1}} & \frac{N B_{b} r_{b}}{m_{1}} & 0 & 0 \\
\frac{K_{t}}{m_{1} r_{b}} & \frac{-\left(m_{1}+m_{2}\right) N K_{b}}{m_{1} m_{2}} & 0 & 0 & \frac{B_{t}}{m_{1} r_{b}} & \frac{-\left(m_{1}+m_{2}\right) N B_{b}}{m_{1} m_{2}} & 0 & 0 \\
0 & 0 & \frac{-K_{s}}{I_{r}} & \frac{K_{s}}{I_{r}} & 0 & 0 & \frac{-B_{s}}{I_{r}} & \frac{B_{s}}{I_{r}} \\
0 & 0 & \frac{K_{s}}{I_{g}} & \frac{-K_{s}}{I_{g}} & 0 & 0 & \frac{B_{s}}{I_{g}} & \frac{-B_{s}}{I_{g}}
\end{array}\right]
$$




$$
\begin{aligned}
& B=\left[\begin{array}{ccc}
0 & 0 & 0 \\
0 & 0 & 0 \\
0 & 0 & 0 \\
0 & 0 & 0 \\
\frac{1}{3 m_{1}} & 0 & 0 \\
\frac{2 m_{1}-m_{2}}{3 m_{1} m_{2} r_{b}} & 0 & 0 \\
0 & \frac{1}{I_{r}} & 0 \\
0 & 0 & \frac{-1}{I_{g}}
\end{array}\right] \\
& C=\left[\begin{array}{llllllll}
0 & 0 & 0 & 0 & 1 & 0 & 0 & 0 \\
0 & 0 & 0 & 0 & 0 & 1 & 0 & 0 \\
0 & 0 & 0 & 0 & 0 & 0 & 1 & 0 \\
0 & 0 & 0 & 0 & 0 & 0 & 0 & 1
\end{array}\right]
\end{aligned}
$$

Building on the work done by Garcia-Sanz and Houpis, Wheeler finds the transfer matrix $\boldsymbol{T}(\mathrm{s})$ between the input $\boldsymbol{u}(\mathrm{s})$ and the output $\boldsymbol{y}(\mathrm{s}) . \boldsymbol{T}(\mathrm{s})$ can be found from the State Space model calculated above, $\boldsymbol{T}(\mathrm{s})=\boldsymbol{C}(\mathrm{s} \boldsymbol{I}-\boldsymbol{A})^{-1} \boldsymbol{B}$.

$$
\boldsymbol{T}(\mathrm{s})=\left[\begin{array}{ccc}
\mu_{11}(s) & 0 & 0 \\
\mu_{21}(s) & 0 & 0 \\
0 & \mu_{32}(s) & \mu_{33}(s) \\
0 & \mu_{42}(s) & \mu_{43}(s)
\end{array}\right]
$$

where

$$
\begin{aligned}
& \mu_{i j}(s)=\frac{n_{\mu i j}(s)}{d_{\mu i j}(s)}, \quad i=1,2,3,4 ; j=1,2,3 \\
& n_{\mu 11}(s)=m_{2} s^{3}+3 N B_{b} s^{2}+3 N K_{b} s
\end{aligned}
$$




$$
\begin{aligned}
& d_{\mu 11}(s)=3 m_{1} m_{2} s^{4}+\left(3 N B_{b} m_{2}+3 B_{t} m_{2}+3 N B_{b} m_{1}\right) s^{3}+\left(3 K_{t} m_{2}+3 B_{t} N B_{b}+3 N K_{b} m_{1}+3 N K_{b} m_{2}\right) s^{2} \\
& n_{\mu 21}(s)=\left(2 m_{1}-m_{2}\right) s^{3}+2 B_{t} s^{2}+2 K_{t} s \\
& d_{\mu 21}(s)=r_{b} d_{\mu 11}(s) \\
& n_{\mu 32}(s)=I_{g} s^{2}+B_{s} s+K_{s} \\
& d_{\mu 32}(s)=I_{r} I_{g} s^{3}+\left(I_{r} B_{s}+I_{g} B_{s}\right) s^{2}+\left(I_{r} K_{s}+I_{g} K_{s}\right) s+3 N K_{b} K_{t}(23) \\
& n_{\mu 33}(s)=-\left(B_{s} s+K_{s}\right) \\
& d_{\mu 33}(s)=d_{\mu 32}(s) \\
& n_{\mu 42}(s)=B_{s} s+K_{s} \\
& d_{\mu 42}(s)=d_{\mu 32}(s) \\
& n_{\mu 43}(s)=-\left(I_{r} s^{2}+B_{s} s+K_{s}\right) \\
& d_{\mu 43}(s)=d_{\mu 32}(s)
\end{aligned}
$$$$
+\left(3 B_{t} N K_{b}+3 N B_{b} K_{t}\right) s+3 N K_{b} K_{t}(23)
$$

The aerodynamic equations show that $\mathrm{F}_{\mathrm{T}}$ and $\mathrm{T}_{\mathrm{r}}$, depend nonlinearly on $\mathrm{v} 1, \beta$, and $\Omega_{1}$.

The aerodynamic equations:

$$
\begin{aligned}
& F_{T}=0.5 \rho \pi r_{b}^{2} C_{T}(\lambda, \beta) v_{1}^{2} \\
& T_{r}=\rho \pi r_{b}^{2} C_{P}(\lambda, \beta) v_{1}^{3}
\end{aligned}
$$

In the equations above, $\mathrm{C}_{\mathrm{p}}$ is the power coefficient, the ratio of the mechanical power extracted by the wind turbine to the original power of the free-air stream that flows through the rotor cross-sectional area [8]. $\mathrm{C}_{\mathrm{T}}$ is the thrust coefficient. a represents the axial induction factor, a measure of how much of the air's velocity is lost when it passes 
through the wind turbine rotor. It can be written as $0.5\left(1-\mathrm{v}_{2} / \mathrm{v}_{1}\right)$, a function of the far upstream air velocity v1 and the downstream air velocity v2. The equations for the power and thrust coefficients are given below.

$C_{T}(\lambda, \beta)=4 a(1-a)$

and

$C_{P}(\lambda, \beta)=0.39\left(\frac{116}{\lambda_{i}}-0.4 \beta-5\right) e^{\frac{-16.5}{\lambda_{i}}}$

where

$$
\lambda_{i}=\left(\frac{1}{\lambda+0.089 \beta}-\frac{0.035}{\beta^{3}+1}\right)^{-1}
$$

$\lambda$ is the tip speed ratio, or the ratio of the product of the rotor speed and the blade radius and the wind velocity. $\beta$ is the pitch angle. $\rho$ describes the air density [8].

When the aerodynamics equations are linearized around a working point ( $\mathrm{v} 10,_{0}$, $\Omega_{\mathrm{r} 0}$ ), and the bias components are ignored, the transfer matrix that describes the inputs FT and $T_{r}$ is composed only of gain elements.

$$
\left[\begin{array}{c}
F_{T}(s) \\
T_{r}(s)
\end{array}\right]=\left[\begin{array}{lll}
K_{F \Omega} & K_{F V} & K_{F \beta} \\
K_{T \Omega} & K_{T V} & K_{T \beta}
\end{array}\right]\left[\begin{array}{c}
\Omega_{r}(s) \\
v_{1}(s) \\
\beta(s)
\end{array}\right]
$$

The gains can be calculated using the $C_{p}$ curves and equations $40-45$ below [8]. The $C_{p}$ curves are shown in Appendix A.

$$
\begin{aligned}
& K_{F \Omega}=\left.\frac{\partial F_{T}(t)}{\partial \Omega_{r}(t)}\right|_{0}=\left.\frac{1}{2} \rho \pi r_{b}^{2} \frac{\partial C_{T}}{\partial \Omega_{r}}\right|_{0} v_{10}^{2} \\
& K_{F V}=\left.\frac{\partial F_{T}(t)}{\partial v_{1}(t)}\right|_{0}=\frac{1}{2} \rho \pi r_{b}^{2}\left[\left.\frac{\partial C_{T}}{\partial \Omega_{r}}\right|_{0} v_{10}^{2}+2 v_{10} C_{r 0}\right]
\end{aligned}
$$




$$
\begin{aligned}
& K_{F \beta}=\left.\frac{\partial F_{T}(t)}{\partial \beta(t)}\right|_{0}=\left.\frac{1}{2} \rho \pi r_{b}^{2} \frac{\partial C_{T}}{\partial \beta}\right|_{0} v_{10}^{2} \\
& K_{T \Omega}=\left.\frac{\partial T_{r}(t)}{\partial \Omega_{r}(t)}\right|_{0}=\frac{1}{2} \rho \pi r_{b}^{2}\left[\left.\frac{\partial C_{P}}{\partial \Omega_{r}}\right|_{0} \frac{1}{\Omega_{r 0}}-C_{P 0} \frac{1}{\Omega_{r 0}^{2}}\right] v_{10}^{3} \\
& K_{T V}=\left.\frac{\partial T_{r}(t)}{\partial v_{1}(t)}\right|_{0}=\frac{1}{2} \rho \pi r_{b}^{2} \frac{1}{\Omega_{r 0}}\left[\left.\frac{\partial C_{P}}{\partial v_{1}}\right|_{0} v_{10}^{3}+3 C_{P 0} v_{10}^{2}\right] \\
& K_{T \beta}=\left.\frac{\partial T_{r}(t)}{\partial \beta(t)}\right|_{0}=\left.\frac{1}{2} \rho \pi r_{b}^{2} \frac{1}{\Omega_{r 0}} \frac{\partial C_{P}}{\partial \beta}\right|_{0} v_{10}^{3}
\end{aligned}
$$

Combining the linearized description of the aerodynamics with the transfer matrix, Wheeler obtains the overall wind turbine transfer matrix.

$$
\left[\begin{array}{l}
y_{t}^{\prime}(s) \\
\gamma^{\prime}(s) \\
\Omega_{r}(s) \\
\Omega_{g}(s)
\end{array}\right]=P^{\prime}(s)\left[\begin{array}{c}
\beta(s) \\
T_{g}(s)
\end{array}\right]+D^{\prime}(s) \mathrm{v}_{1}(s)
$$

where

$$
P^{\prime}(\mathrm{s})=\left[\begin{array}{cc}
\mu_{11}(s) \frac{\mu_{32}(s)\left(K_{F \Omega} K_{T \beta}-K_{F \beta} K_{T \Omega}\right)+K_{F \beta}}{1-\mu_{32}(s) K_{T \Omega}} & \mu_{11}(s) \frac{\mu_{33}(s) K_{F \Omega}}{1-\mu_{32}(s) K_{T \Omega}} \\
\mu_{21}(s) \frac{\mu_{32}(s)\left(K_{F \Omega} K_{T \beta}-K_{F \beta} K_{T \Omega}\right)+K_{F \beta}}{1-\mu_{32}(s) K_{T \Omega}} & \mu_{21}(s) \frac{\mu_{33}(s) K_{F \Omega}}{1-\mu_{32}(s) K_{T \Omega}} \\
\mu_{32}(s) \frac{K_{T \beta}}{1-\mu_{32}(s) K_{T \Omega}} & \mu_{33}(s) \frac{1}{1-\mu_{32}(s) K_{T \Omega}} \\
\mu_{42}(s) \frac{K_{T \beta}}{1-\mu_{32}(s) K_{T \Omega}} & \frac{\mu_{42}(s) \mu_{33}(s) K_{T \Omega}+\mu_{43}(s)-\mu_{43}(s) \mu_{32}(s) K_{T \Omega}}{1-\mu_{32}(s) K_{T \Omega}}
\end{array}\right]
$$




$$
D^{\prime}(s)=\left[\begin{array}{c}
\mu_{11}(s) \frac{\mu_{32}(s)\left(K_{F \Omega} K_{T V}-K_{F V} K_{T \Omega}\right)+K_{F V}}{1-\mu_{32}(s) K_{T \Omega}} \\
\mu_{21}(s) \frac{\mu_{32}(s)\left(K_{F \Omega} K_{T V}-K_{F V} K_{T \Omega}\right)+K_{F V}}{1-\mu_{32}(s) K_{T \Omega}} \\
\mu_{32}(s) \frac{K_{T V}}{1-\mu_{32}(s) K_{T \Omega}} \\
\mu_{42}(s) \frac{K_{T V}}{1-\mu_{32}(s) K_{T \Omega}}
\end{array}\right]
$$

The transfer functions of the actuators are

$$
\begin{aligned}
& \beta(s)=A_{\beta}(s) \beta_{d}(s) \\
& T_{g}(s)=A_{T}(s) T_{g d}(s)
\end{aligned}
$$

In the equations above, $\beta_{\mathrm{d}}$ is the demanded pitch angle and $\mathrm{T}_{\mathrm{gd}}$ is the demanded electrical torque (both calculated by the control system). $A_{\beta}(\mathrm{s})$ and $\mathrm{A}_{\mathrm{T}}(\mathrm{s})$ are the transfer functions from the control signals $\beta_{\mathrm{d}}$ and $\mathrm{T}_{\mathrm{gd}}$ to the actual values of the actuators [8]. Combining these equations with the system above results in the following equation:

$$
\begin{aligned}
& {\left[\begin{array}{c}
y_{t}^{\prime}(s) \\
\gamma^{\prime}(s) \\
\Omega_{r}(s) \\
\Omega_{g}(s)
\end{array}\right]=P(s)\left[\begin{array}{c}
\beta_{d}(s) \\
T_{g d}(s)
\end{array}\right]+D(s) \mathrm{v}_{1}(s)} \\
& P(s)=\left[\begin{array}{cc}
\mu_{11}(s) \frac{\mu_{32}(s)\left(K_{F \Omega} K_{T \beta}-K_{F \beta} K_{T \Omega}\right)+K_{F \beta}}{1-\mu_{32}(s) K_{T \Omega}} A_{\beta}(s) & \mu_{11}(s) \frac{\mu_{33}(s) K_{F \Omega}}{1-\mu_{32}(s) K_{T \Omega}} A_{T}(s) \\
\mu_{21}(s) \frac{\mu_{32}(s)\left(K_{F \Omega} K_{T \beta}-K_{F \beta} K_{T \Omega}\right)+K_{F \beta}}{1-\mu_{32}(s) K_{T \Omega}} A_{\beta}(s) & \mu_{21}(s) \frac{\mu_{33}(s) K_{F \Omega}}{1-\mu_{32}(s) K_{T \Omega}} A_{T}(s) \\
\mu_{32}(s) \frac{K_{T \beta}}{1-\mu_{32}(s) K_{T \Omega}} A_{\beta}(s) & \mu_{33}(s) \frac{1}{1-\mu_{32}(s) K_{T \Omega}} A_{T}(s) \\
\mu_{42}(s) \frac{K_{T \beta}}{1-\mu_{32}(s) K_{T \Omega}} A_{\beta}(s) & \frac{\mu_{42}(s) \mu_{33}(s) K_{T \Omega}+\mu_{43}(s)-\mu_{43}(s) \mu_{32}(s) K_{T \Omega}}{1-\mu_{32}(s) K_{T \Omega}} A_{T}(s)
\end{array}\right]
\end{aligned}
$$




$$
D(s)=\left[\begin{array}{c}
\mu_{11}(s) \frac{\mu_{32}(s)\left(K_{F \Omega} K_{T V}-K_{F V} K_{T \Omega}\right)+K_{F V}}{1-\mu_{32}(s) K_{T \Omega}} \\
\mu_{21}(s) \frac{\mu_{32}(s)\left(K_{F \Omega} K_{T V}-K_{F V} K_{T \Omega}\right)+K_{F V}}{1-\mu_{32}(s) K_{T \Omega}} \\
\mu_{32}(s) \frac{K_{T V}}{1-\mu_{32}(s) K_{T \Omega}} \\
\mu_{42}(s) \frac{K_{T V}}{1-\mu_{32}(s) K_{T \Omega}}
\end{array}\right]
$$

where $\boldsymbol{P}(\mathrm{s})$ is the plant matrix and $\boldsymbol{D}(\mathrm{s})$ is the disturbance transfer matrix [28].

The above matrices describe the entire direct drive wind turbine.

The model for collective pitch controller requires only the equation for the rotational speed, $\Omega_{\mathrm{r}}$. The rotational speed depends on demanded control signals for the blade pitch angle and the wind speed [8]. This is shown in the equation below:

$\Omega_{r}(s)=\frac{1}{1-\mu_{32}(s) K_{T \Omega}}\left\{\mu_{32}(s)\left[K_{T \beta} A_{\beta}(s) \beta_{d}(s)+K_{T V} v_{1}(s)\right]+\mu_{33}(s) A_{T}(s) T_{g d}(s)\right\}$

This can be expressed as:

$\Omega_{r}(s)=F_{1}(s) v_{1}(s)+F_{2}(s) \beta_{d}(s)+F_{3}(s) T_{g d}(s)$

where

$$
\begin{aligned}
& F_{1}(s)=\frac{K_{T V} n_{\mu 32}(s)}{d_{t f}(s)} \\
& F_{2}(s)=\frac{K_{T \beta} n_{\mu 32}(s) A_{\beta}(s)}{d_{t f}(s)} \\
& F_{3}(s)=\frac{n_{\mu 33}(s) A_{T}(s)}{d_{t f}(s)} \\
& n_{\mu 32}(s)=I_{g} s^{2}+B_{s} s+K_{s} \\
& n_{\mu 33}(s)=-\left(B_{s} s+K_{s}\right)
\end{aligned}
$$


$d_{t f}(s)=d_{\mu 32}(s)-n_{\mu 32}(s) K_{T \Omega}=I_{r} I_{g} s^{3}+\left(I_{r} B_{s}+I_{g} B_{s}-I_{g} K_{T \Omega}\right) s^{2}+\left(K_{s} I_{g}+I_{r} K_{s}-B_{s} K_{T \Omega}\right) s-K_{s} K_{T \Omega}$

The block diagram of the system is shown below:

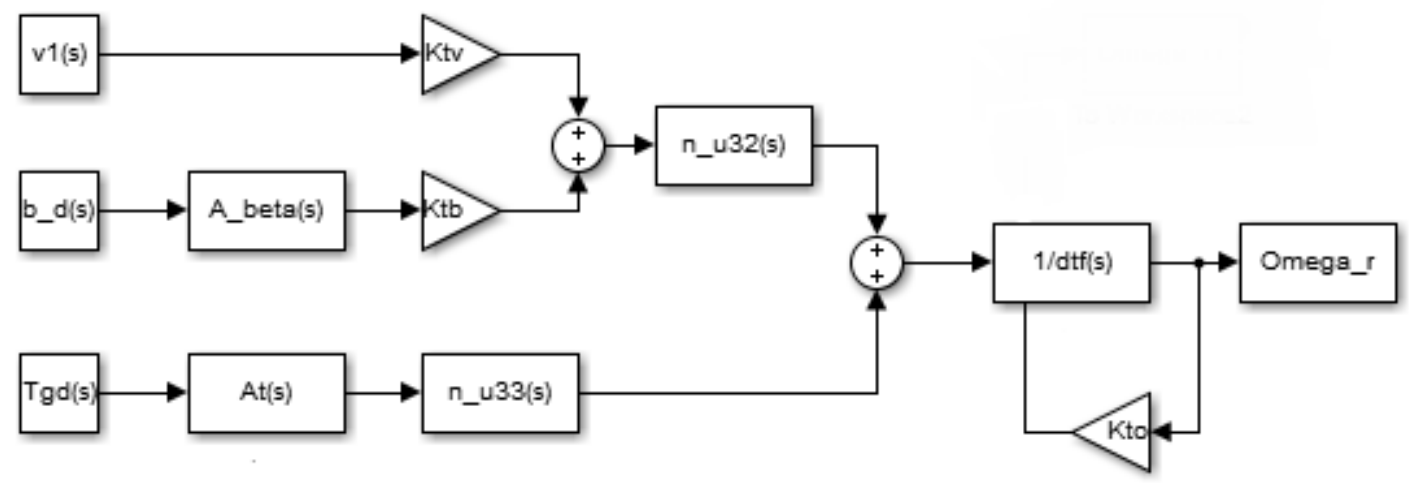

Figure 4.1: Detailed Block Diagram of the Collective Pitch Control Systems

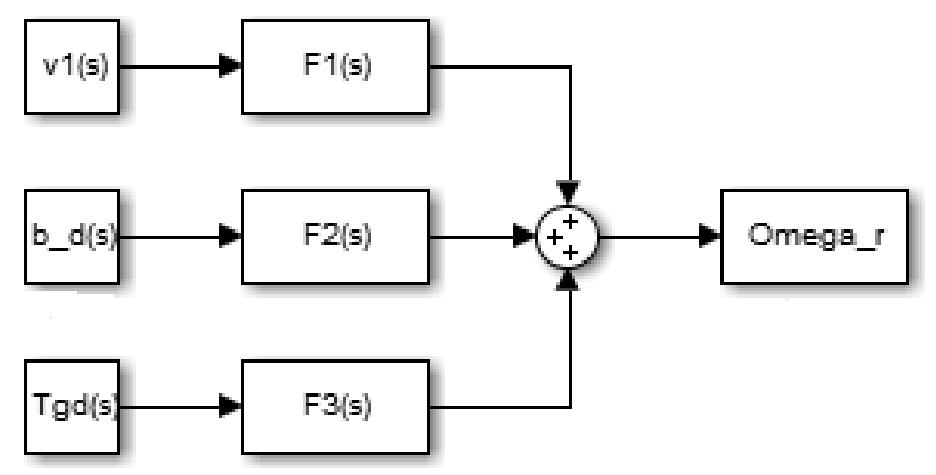

Figure 4.2: Reduced Block Diagram of the Collective Pitch Control Systems

The values of the parameters used in the model for the collective pitch control system are given by Jonkman and Jonkman et al in NRELOffshrBsline5MW and Definition of a 5-MW Reference Wind Turbine for Offshore System Development and

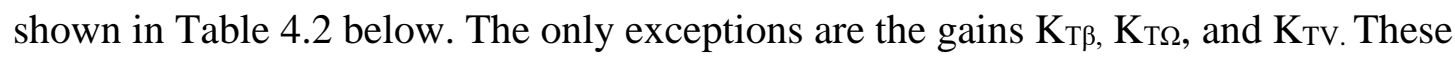
gains are found using the Simulink model shown in Figure 4.3, where the lookup table contains the data for $\mathrm{Cp} / \lambda$ curves shown in Appendix A. First, the working points for 
each wind speed from 14 to $20 \mathrm{~m} / \mathrm{s}$ are found. The working point is the combination of wind speed and pitch angle that leads to the system running at the rated rotor speed of $1.27 \mathrm{rad} / \mathrm{s}$. The working points are shown in Table 4.1.

\begin{tabular}{|c|c|c|c|}
\hline $\begin{array}{c}\text { Rotor speed } \\
\Omega(\mathrm{rad} / \mathrm{s})\end{array}$ & $\begin{array}{c}\text { Wind speed } \\
\mathrm{v}(\mathrm{m} / \mathrm{s})\end{array}$ & $\begin{array}{c}\text { Pitch angle } \\
\beta(\text { degrees })\end{array}$ & $\begin{array}{c}\text { Electrical torque } \\
\mathrm{Tg}(\mathrm{Nm})\end{array}$ \\
\hline 1.27 & 14 & 8.4 & 43094 \\
\hline 1.27 & 15 & 10.2 & 43094 \\
\hline 1.27 & 16 & 11.8 & 43094 \\
\hline 1.27 & 17 & 13.3 & 43094 \\
\hline 1.27 & 18 & 14.7 & 43094 \\
\hline 1.27 & 19 & 16.0 & 43094 \\
\hline 1.27 & 20 & 17.3 & 43094 \\
\hline
\end{tabular}

Table 4.1: Working Points for Collective Pitch Control Model
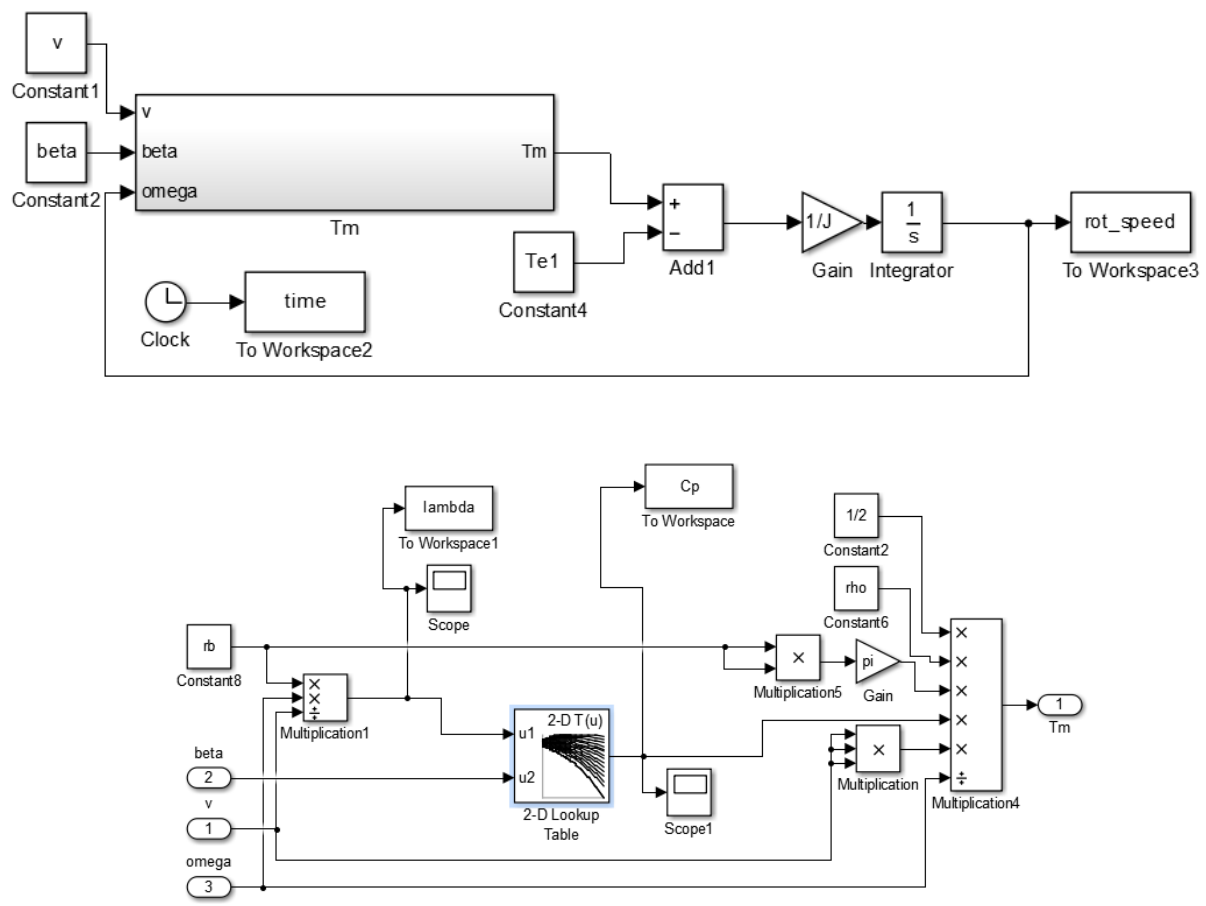

Figure 4.3: Simulink Model Used to Find Working Points 
Then, based on perturbations around the working point, the relationship between the rotor speed and wind speed, pitch angle, and electrical torque can be found. This relationship is shown in equation 62 . Combining that relationship with the steady state values of equations 55-61, the gains $\mathrm{K}_{\mathrm{T} \beta}, \mathrm{K}_{\mathrm{T} \Omega}$, and $\mathrm{K}_{\mathrm{TV}}$ can be found.

$$
\begin{aligned}
& \Omega=K_{1} v+K_{2} \beta+K_{3} T \\
& K_{1}=\frac{-K_{T V}}{K_{T \Omega}} \\
& K_{2}=\frac{-K_{T \beta}}{K_{T \Omega}} \\
& K_{3}=\frac{1}{K_{T \Omega}}
\end{aligned}
$$

The table below shows the values used in this thesis for the parameters [12, 15].

\begin{tabular}{|l|l|l|l|}
\hline Power to the grid & $5.0 \mathrm{MW}$ & $\mathrm{rb}_{\mathrm{b}}$ & $63 \mathrm{~m}$ \\
\hline Density of air & $1.225 \mathrm{~kg} / \mathrm{m}^{3}$ & $\mathrm{~K}_{\mathrm{s}}$ & $8.67 * 10^{8} \mathrm{Nm}$ \\
\hline $\mathrm{N}$ & 3 & $\mathrm{~B}_{\mathrm{s}}$ & $6.22^{*} 10^{6} \mathrm{Nms}$ \\
\hline $\mathrm{I}_{\mathrm{r}}$ & $3.54 * 10^{7} \mathrm{~kg} \mathrm{~m}{ }^{2}$ & $\mathrm{I}_{\mathrm{g}}$ & $5.03^{*} 10^{6} \mathrm{~kg} \mathrm{~m}{ }^{2}$ \\
\hline$\Omega_{\mathrm{r} \_ \text {nom }}$ & $1.27 \mathrm{rad} / \mathrm{s}$ & $\mathrm{K}_{\mathrm{T} \beta}$ nominal & $\left.-4.78^{*} 10^{7} \mathrm{Nm} / \mathrm{rad}\right)$ \\
\hline $\mathrm{K}_{\mathrm{T} \Omega}$ nominal & $-1.11^{*} 10^{7} \mathrm{Nm} /(\mathrm{rad} / \mathrm{s})$ & $\mathrm{K}_{\mathrm{TV}}$ nominal & $1.72 * 10^{6} \mathrm{Nm} /(\mathrm{m} / \mathrm{s})$ \\
\hline $\mathrm{K}_{\mathrm{T} \beta}$ minimum & $-5.73 * 10^{7} \mathrm{Nm} /(\mathrm{rad})$ & $\mathrm{K}_{\mathrm{T} \beta}$ maximum & $-3.05^{*} 10^{7} \mathrm{Nm} /(\mathrm{rad})$ \\
\hline $\mathrm{K}_{\mathrm{T} \Omega}$ minimum & $-1.67 * 10^{7} \mathrm{Nm} /(\mathrm{rad} / \mathrm{s})$ & $\mathrm{K}_{\mathrm{T} \Omega}$ maximum & $-4.67^{*} 10^{6} \mathrm{Nm} /(\mathrm{rad} / \mathrm{s})$ \\
\hline $\mathrm{K}_{\mathrm{TV}}$ minimum & $1.04 * 10^{6} \mathrm{Nm} /(\mathrm{m} / \mathrm{s})$ & $\mathrm{K}_{\mathrm{TV}}$ maximum & $2.13^{*} 10^{6} \mathrm{Nm} /(\mathrm{m} / \mathrm{s})$ \\
\hline AT(s) & 1.0 & $\mathrm{~A}_{\beta}(\mathrm{s})$ & 1.0 \\
\hline
\end{tabular}

Table 4.2: Wind Turbine Parameters for Collective Pitch Control Model 
Note: The parameters above are calculated for the range of wind velocities from 14 to 20 $\mathrm{m} / \mathrm{s}$. The nominal values represent a wind speed of $17 \mathrm{~m} / \mathrm{s}$.

Using the values above,

$$
\begin{aligned}
& n_{\mu 32}(s)=5.03 * 10^{6} s^{2}+6.22 * 10^{6} s+8.67 * 10^{8} \\
& n_{\mu 33}(s)=-6.22 * 10^{6} s-8.67 * 10^{8} \\
& d_{t f}(s)=1.78 * 10^{14} s^{3}+\left(2.51 * 10^{14}-5.03 * 10^{6} K_{T \Omega}\right) s^{2}+\left(3.51 * 10^{16}-6.22 * 10^{6} K_{T \Omega}\right) s-8.67 * 10^{8} K_{T \Omega}
\end{aligned}
$$

The final transfer functions are calculated and given below.

$$
F_{1}(s)=\frac{5.03 * 10^{6} K_{T V} s^{2}+6.22 * 10^{6} K_{T V} s+8.67 * 10^{8} K_{T V}}{1.78 * 10^{14} s^{3}+\left(2.51 * 10^{14}-5.03 * 10^{6} K_{T \Omega}\right) s^{2}+\left(3.51 * 10^{16}-6.22 * 10^{6} K_{T \Omega}\right) s-8.67 * 10^{8} K_{T \Omega}}
$$

$$
F_{2}(s)=\frac{5.03 * 10^{6} K_{T \beta} s^{2}+6.22 * 10^{6} K_{T \beta} s+8.67 * 10^{8} K_{T \beta}}{1.78 * 10^{14} s^{3}+\left(2.51 * 10^{14}-5.03 * 10^{6} K_{T \Omega}\right) s^{2}+\left(3.51 * 10^{16}-6.22 * 10^{6} K_{T \Omega}\right) s-8.67 * 10^{8} K_{T \Omega}}
$$

$$
F_{3}(s)=\frac{-6.22 * 10^{6} s-8.67 * 10^{8}}{1.78 * 10^{14} s^{3}+\left(2.51 * 10^{14}-5.03 * 10^{6} K_{T \Omega}\right) s^{2}+\left(3.51 * 10^{16}-6.22 * 10^{6} K_{T \Omega}\right) s-8.67 * 10^{8} K_{T \Omega}}
$$

\subsection{Controller Design}

The controller design for the collective pitch controller is performed using QFTCT in Matlab. After the three system plants $F_{1}(s), F_{2}(s)$, and $F_{3}(s)$ are entered into the plant definition window, the frequencies of interest are found from the Bode plots of the nominal plant. The Bode plots are given in Appendix B. The selected frequency vector is: $\omega=\left[\begin{array}{lllllll}0.001 & 0.005 & 0.01 & 0.05 & 0.1 & 0.2 & 0.250 .30 .40 .51510501005001000\end{array}\right] \mathrm{rad} / \mathrm{s}$. 


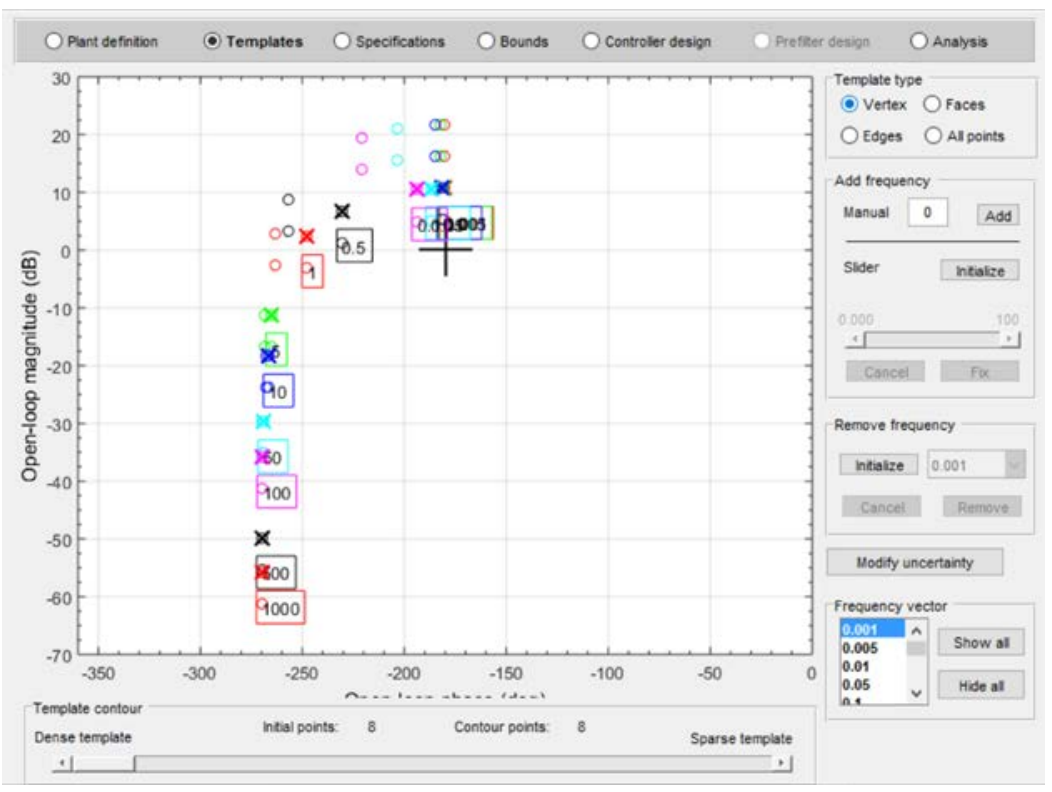

Figure 4.4: Plant Template Window

Next, the design specifications are entered into the specification window. The stability specification selected is $\mathrm{W}_{\mathrm{s}}=1.21$, which translates to a gain margin of $5.23 \mathrm{~dB}$ and a phase margin of $48.8^{\circ}$. The user-defined disturbance rejection specification is given in equation 72 below.

$\left|\frac{F_{1}(j \omega)}{1+P(j \omega) C(j \omega)}\right| \leq\left|\frac{0.43 j \omega}{0.43 j \omega+1}\right|$

The specification bounds window from QFTCT for collective pitch controller design is given in Figure 4.5. 


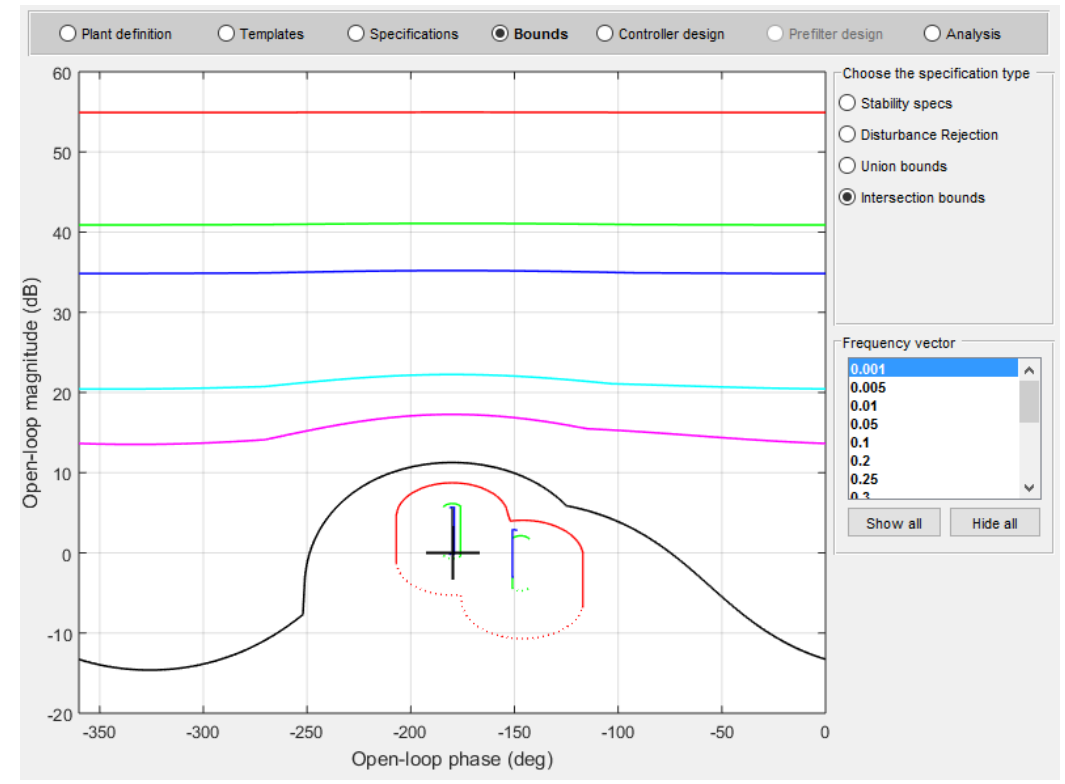

Figure 4.5: Intersection Bounds Window

A PI controller is selected as the form of the collective pitch controller. The equation of the selected controller is given below, and the controller design window from QFTCT is shown in Figure 4.6.

$C(s)=\frac{-0.401 s-0.172}{s}$

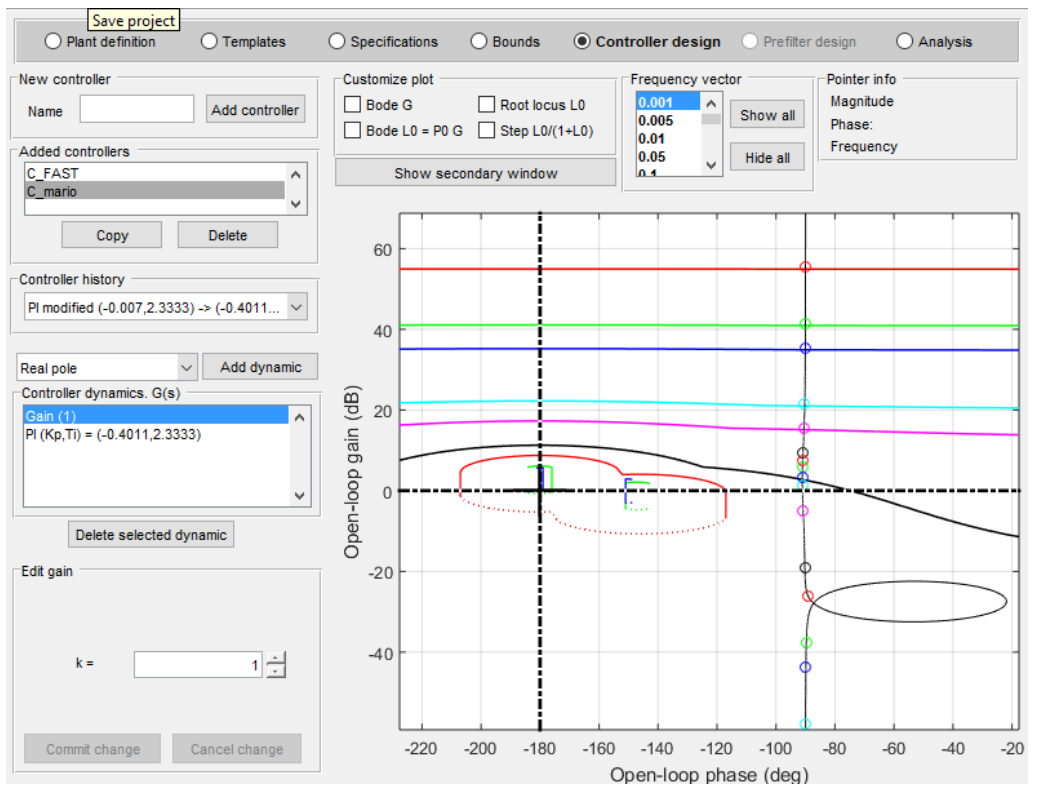

Figure 4.6: Controller Design Window 
This controller is based on the gain-scheduled PI controller given by NREL for the 5MW reference turbine [15]. It meets all QFT bound requirements at the specified frequencies as seen in the QFTCT analysis windows below.

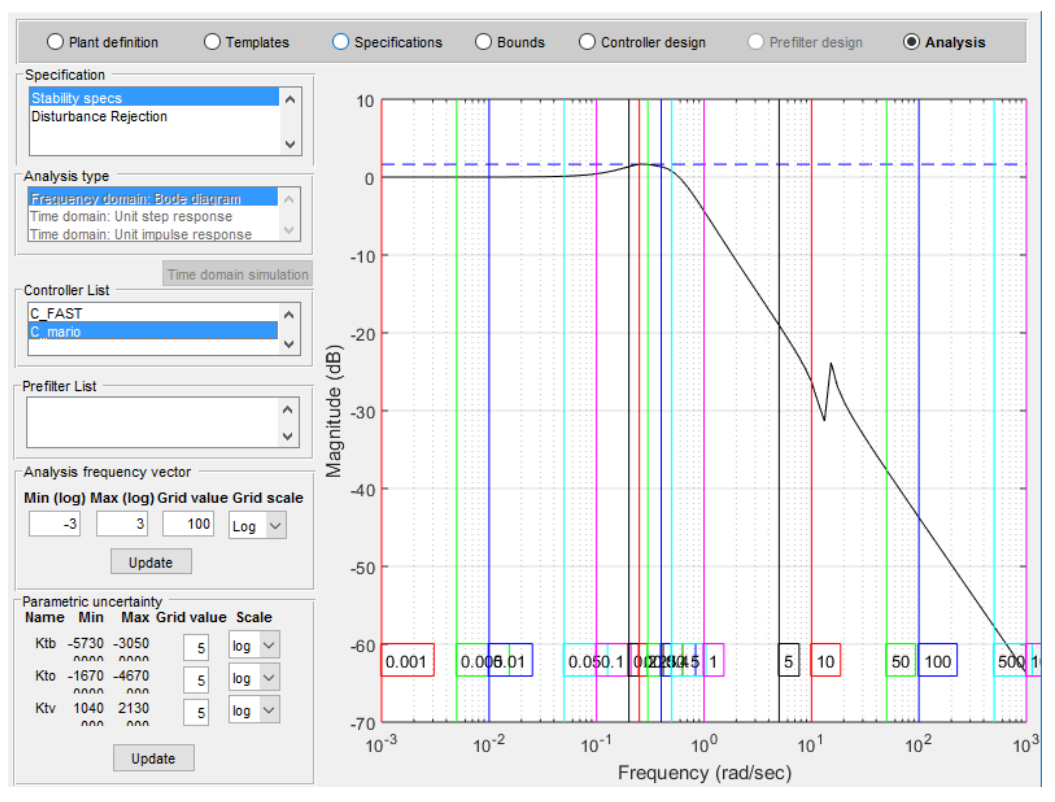

Figure 4.7: Stability Analysis Window

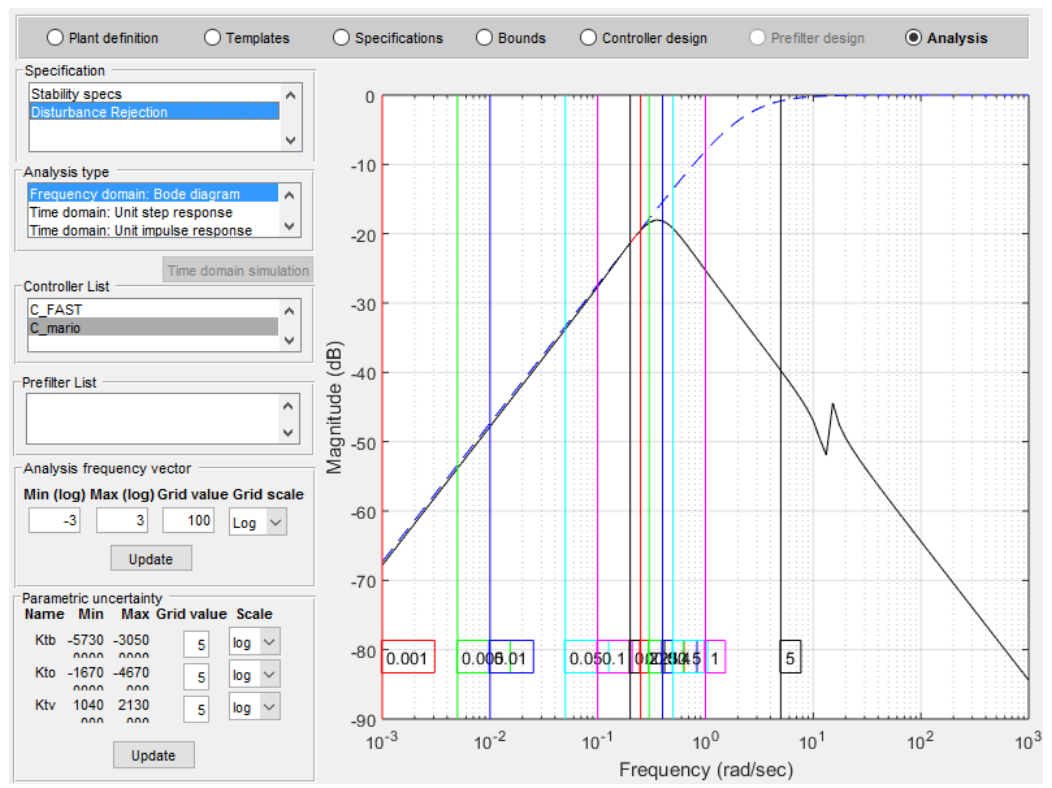

Figure 4.8: Disturbance Rejection Analysis Window 


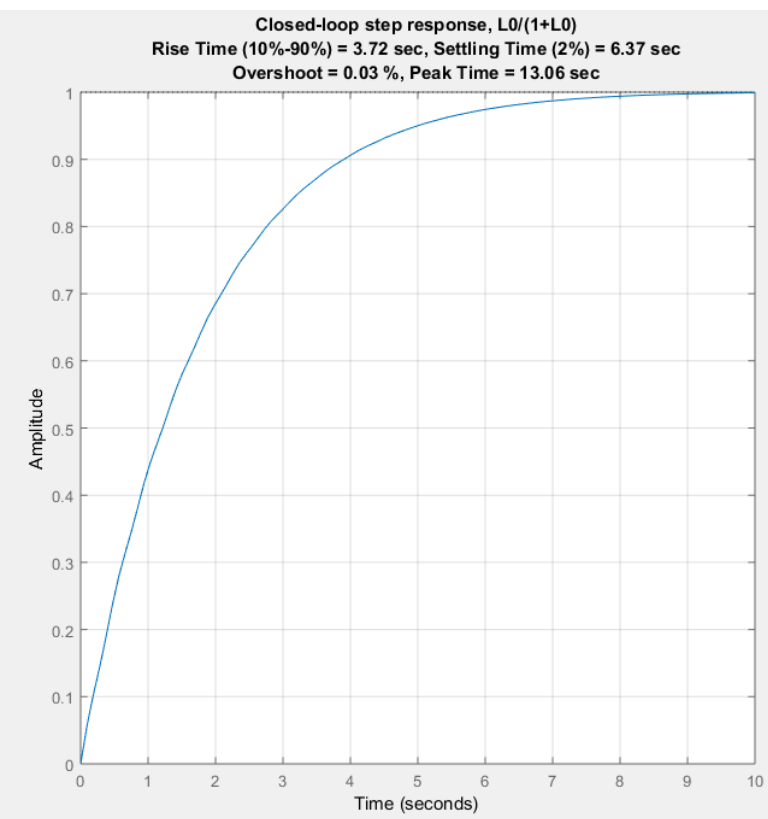

Figure 4.9: Step Response (Secondary Analysis Window) 


\section{Chapter 5 - Individual Pitch Control}

\subsection{Model}

There are several models for individual pitch control presented in the literature. An overwhelming majority of these models use the Coleman transformation. The Coleman transformation (also called the multi-blade coordinate blade transformation) comes from the field of helicopter control [17]. The use of the Coleman transformation simplifies the design process by decoupling the symmetric and asymmetric parts of the blade's equation of motion. It transforms the equations of motion from the rotating coordinate frame to fixed coordinate frames $[17,27]$. The fixed coordinate frames are often referred to as the tilt and yaw coordinate frames. This decoupling allows the loads on the non-rotating parts of the turbine to be examined and then reduced with the individual pitch controller.

The forward Coleman transformation is given in equation 74 , and the inverse transformation is given in equation 75 .

$$
\begin{aligned}
& {\left[\begin{array}{l}
\theta_{1}(t) \\
\theta_{2}(t) \\
\theta_{3}(t)
\end{array}\right]=\left[\begin{array}{ccc}
1 & \cos \phi(t) & \sin \phi(t) \\
1 & \cos \left(\phi(t)+\frac{2 \pi}{3}\right) & \sin \left(\phi(t)+\frac{2 \pi}{3}\right) \\
1 & \cos \left(\phi(t)+\frac{4 \pi}{3}\right) & \sin \left(\phi(t)+\frac{4 \pi}{3}\right)
\end{array}\right]\left[\begin{array}{c}
\bar{\theta}(t) \\
\theta_{\text {tilt }}(t) \\
\theta_{\text {yaw }}(t)
\end{array}\right]} \\
& {\left[\begin{array}{c}
-\bar{M}(t) \\
M_{\text {tilt }}(t) \\
M_{\text {yaw }}(t)
\end{array}\right]=\left[\begin{array}{ccc}
\frac{1}{3} & \frac{1}{3} \\
\frac{2}{3} \cos \phi(t) & \frac{2}{3} \cos \left(\phi(t)+\frac{2 \pi}{3}\right) & \frac{2}{3} \cos \left(\phi(t)+\frac{4 \pi}{3}\right) \\
\frac{2}{3} \sin \phi(t) & \frac{2}{3} \sin \left(\phi(t)+\frac{2 \pi}{3}\right) & \frac{2}{3} \sin \left(\phi(t)+\frac{4 \pi}{3}\right)
\end{array}\right]\left[\begin{array}{l}
\frac{1}{3} \\
M_{2}(t) \\
M_{3}(t)
\end{array}\right]}
\end{aligned}
$$


In the equations above, $\theta_{\mathrm{i}}(\mathrm{t})$ and $\mathrm{M}_{\mathrm{i}}(\mathrm{t})$ represent the total pitch angle demands and the total blade root flap-wise bending moments for blades 1, 2, and 3. $\theta_{\text {tilt,yaw }}(\mathrm{t})$ and $\mathrm{M}_{\text {tilt,yaw }}(\mathrm{t})$ are the total pitch angles and blade root flap-wise bending moments in the tilt and yaw coordinate frames, respectively. $\overline{\theta(t)}$ and $\overline{\mathbf{M}^{-}(t)}$ represent the averaged blade-pitch angle demand and the averaged flap-wise bending moment.

The total pitch angle for each blade is the sum of the averaged pitch angle and the perturbations in each blade’s pitch angle demand. The total blade root flap-wise bending moment for each blade is the sum of the averaged bending moment and the perturbations in each blade's bending moment. $\phi(\mathrm{t})$ is the rotor azimuth angle, which is calculated as the product of the rotor speed and time. These relations are expressed below.

$$
\begin{aligned}
& {\left[\begin{array}{l}
\theta_{1}(t) \\
\theta_{2}(t) \\
\theta_{3}(t)
\end{array}\right]=\left[\begin{array}{l}
\bar{\theta}(t)+\tilde{\theta_{1}(t)} \\
\bar{\theta}(t)+\tilde{\theta_{2}}(t) \\
\bar{\theta}(t)+\tilde{\theta_{3}}(t)
\end{array}\right]} \\
& {\left[\begin{array}{l}
M_{1}(t) \\
M_{2}(t) \\
M_{3}(t)
\end{array}\right]=\left[\begin{array}{l}
\bar{M}(t)+\tilde{M_{1}}(t) \\
\bar{M}(t)+\tilde{M}_{2}(t) \\
\bar{M}(t)+\tilde{M_{3}}(t)
\end{array}\right]} \\
& \phi(t)=\Omega_{r} * t
\end{aligned}
$$

Using the Coleman transformation, the root flapwise bending moments can be transformed into bending moments in the tilt and yaw coordinate system, respectively. These two bending moments are the input into the individual pitch controller, and the tilt and yaw referred pitch angles are the individual pitch controller output. Then, the inverse 
Coleman transformation is used to transform the pitch angles in the tilt and yaw coordinate systems into the commanded pitch angles for each blade.

As shown above using the Coleman transformation, the individual pitch controller input is the bending moments on the blades, which comes from the wind turbine. The output from the controller to the turbine is the demanded pitch angle. Therefore, the individual pitch controller requires both sensors to record controller input and actuators to implement the controller output. In the individual pitch controller system, the actuators are clearly the three independent motors used to change the pitch angle of each blade.

For sensors, there are at least two options to record the bending moments on the blades. A strain gauge to measure the deformation of the blade can be located in the root of each blade. This type of sensor is easy to implement, but may not be accurate enough. Alternatively, a distributed fiber optic sensor can be integrated throughout the entire length of the blade to record the displacements that occur along the blade. While the fiber optic sensors are able to gather more information on the loads on the blades than the strain gauge sensors, they are much more difficult to implement. This is because the fiber optic sensors must be built in the blades during the manufacturing process. In either case, the system will have three independent actuators and three independent sensors.

Additionally, the relationship between the tilt and yaw bending moments and the tilt and yaw referred pitch angles is required for the individual pitch controller design. Many of the models used in the existing research on individual pitch control are time domain models. These models are not ideal as the goal of individual pitch control is to reduce loads on the wind turbine at specific frequencies, a specification that is much more easily specified in the frequency domain than in the time domain. Lu et al. and Vali 
et al. both present frequency domain models based on the frequency domain representation of the Coleman transformation and the transfer function that relates the change in root flapwise bending moment to the change in blade pitch angle demands.

Both of these models were considered as potential plants for the individual pitch controller. However, both models were rejected as simulation results showed neither model accurately reflected the behavior of the $5 \mathrm{MW}$ reference turbine in FAST. Therefore, the relationship between the bending moments and pitch angles in the tilt and yaw coordinate frames was determined experimentally. The model for this determination is shown in Figures 5.1 and 5.2.

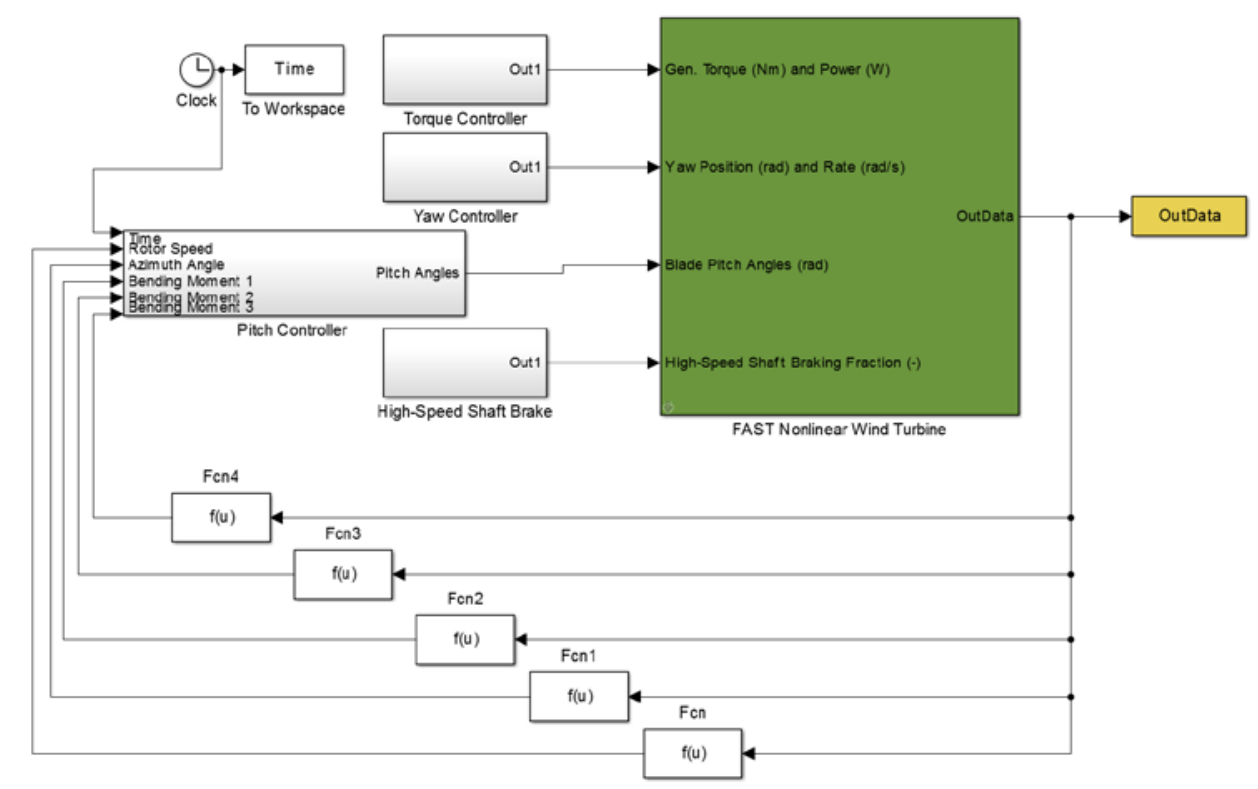

Figure 5.1: Step Simulation to Find Individual Pitch Control Model

To find the plant for the individual pitch controller, the simulation was run with a constant wind speed of $17 \mathrm{~m} / \mathrm{s}$. In the first ten seconds of the simulation, the collective pitch controller and a simple torque controller run to ensure the wind turbine is operating at the working point. At 10 seconds the individual pitch control begins, and a step input is

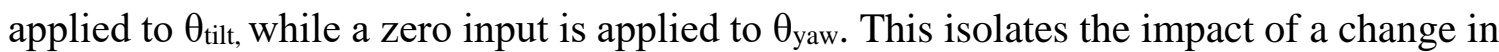


$\theta_{\text {tilt }}$ on $\mathbf{M}_{\text {tilt }}$ and $\mathbf{M}_{\text {yaw. }}$ Then the simulation experiment is repeated with a step input at $\theta_{\text {yaw }}$ and zero input at $\theta_{\text {tilt, }}$ which isolates the relationship between $\theta_{\text {yaw }}$ and $\mathrm{Myw}_{\text {yaw }}$ and the relationship between $\theta_{\text {yaw }}$ and $\mathrm{M}_{\text {tilt. }}$

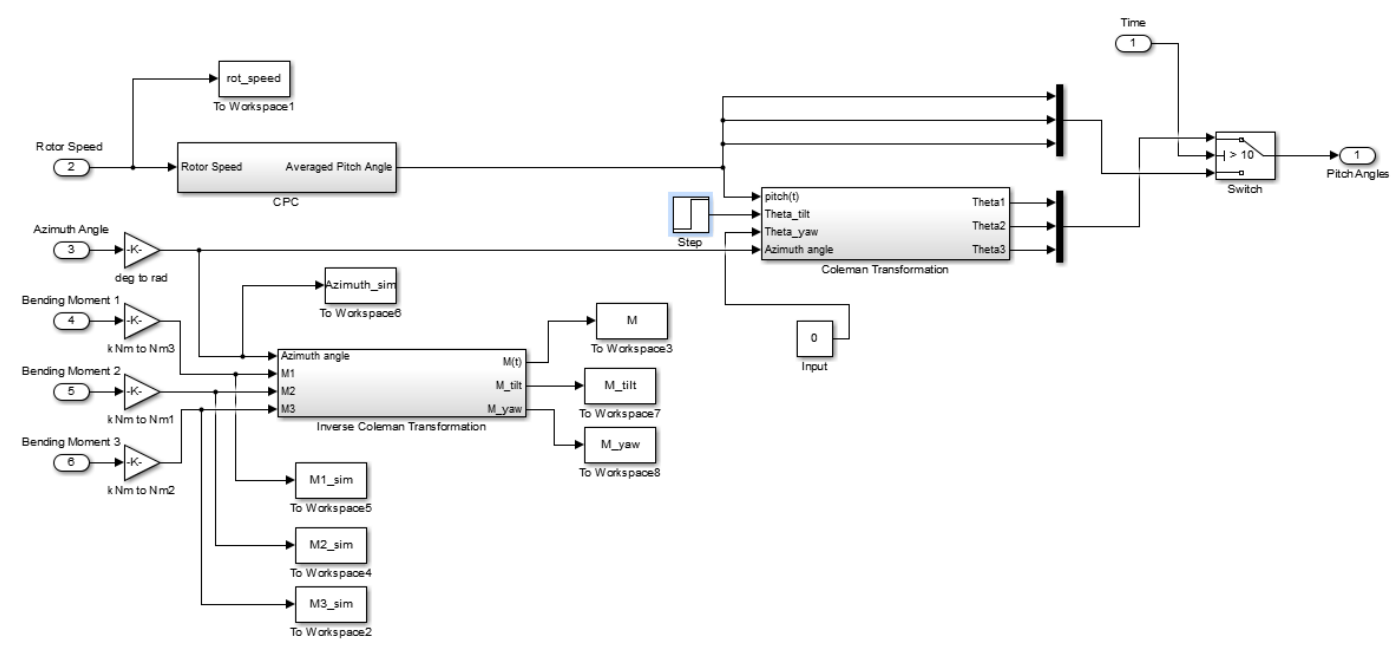

Figure 5.2: Pitch Control Block for Step Simulation

The simulation experiment showed that the relationship between bending moments and demanded pitch angles in the tilt and yaw coordinate frames is best described by a gain. This is shown in the equation below.

$\left[\begin{array}{c}M_{\text {tilt }} \\ M_{\text {yaw }}\end{array}\right]=\left[\begin{array}{cc}-2.85 * 10^{7} & 1.00 * 10^{6} \\ -1.00 * 10^{6} & -2.75 * 10^{7}\end{array}\right]\left[\begin{array}{c}\theta_{\text {tilt }} \\ \theta_{\text {yaw }}\end{array}\right]$

\subsection{Controller Design}

A single input single output (SISO) controller is designed based on the plant relationships shown in equation 81 below and the desired controller performance in the frequency domain. The goal of the individual pitch controller is to reduce the loads on the non-rotating components of the turbine at the $1 p$ and $3 p$ frequencies. The $1 p$ frequency is the same as the rotational speed of the wind turbine, or $0.2 \mathrm{~Hz}$. The $3 p$ frequency is three times the 1p frequency, or $0.6 \mathrm{~Hz}$. The form of the individual pitch controller is shown in equation 81 below. 


$$
\begin{aligned}
& {\left[\begin{array}{l}
M_{\text {tilt }} \\
M_{\text {yaw }}
\end{array}\right]=\left[\begin{array}{cc}
-2.85 * 10^{7} & 1.00 * 10^{6} \\
-1.00 * 10^{6} & -2.75 * 10^{7}
\end{array}\right]\left[\begin{array}{c}
\theta_{\text {tilt }} \\
\theta_{\text {yaw }}
\end{array}\right]} \\
& \mathrm{G}(s)=\left[\begin{array}{cc}
g_{11}(s) & 0 \\
0 & g_{22}(s)
\end{array}\right]
\end{aligned}
$$

First, a bandpass filter is designed to isolate the dynamics around the $1 \mathrm{p}$ and $3 p$ frequencies. The form of the bandpass filter is shown in equation 82 . To emphasize the $1 p$ and $3 p$ frequencies of the $5 \mathrm{MW}$ reference turbine, $\mathrm{f}_{\mathrm{l}}$ is selected to be $0.2 \mathrm{~Hz}$, and $\mathrm{f}_{\mathrm{h}}$ is selected to be $2 \mathrm{~Hz}$. $\mathrm{k}$ is selected to be the sum of $\mathrm{f}_{\mathrm{h}}$ and $\mathrm{f}_{\mathrm{l}}$ divided by $\mathrm{fl}_{\mathrm{l}}$, which reduces to 11.

$T_{b p}(s)=\frac{2 \pi k f_{l} s}{s^{2}+2 \pi\left(f_{h}+f_{l}\right) s+4 \pi^{2} f_{h} f_{l}}=\frac{4.4 \pi s}{s^{2}+4.4 \pi s+1.6 \pi^{2}}$

Then $g_{11}(\mathrm{~s})$ and $\mathrm{g}_{22}(\mathrm{~s})$ are selected to best meet the above specification while maintaining system stability. In this case, $\mathrm{g}_{11}(\mathrm{~s})$ and $\mathrm{g}_{22}(\mathrm{~s})$ are both selected to be a gain of $-3.0715 * 10^{-8}$.

$G(s)=\left[\begin{array}{cc}-3.0715^{*} 10^{-8} & 0 \\ 0 & -3.0715 * 10^{-8}\end{array}\right]$

Finally, the individual pitch controller is implemented into the FAST simulation model. The bandpass filter filters the tilt and yaw bending moments. Then the filter output is input into the SISO individual pitch controller. A saturation is implemented to limit the perturbation due to the individual pitch control to plus or minus one degree of the pitch angle demanded by the collective pitch controller. Also, initialization switches are included in the pitch control implementation to switch on the individual pitch control at 10 seconds. This gives time for the collective pitch controller to drive the wind turbine into the rated power region of the power curve. 
Relative gain analysis of the plant matrix is performed to determine the likelihood of improvements in system performance when using multi-input multi-output (MIMO) control in place of the SISO controller used above [8]. The equation for the relative gain calculation and the results of the calculation are shown below.

$$
R G A=P(\mathrm{j} \omega) *\left(P^{-1}(\mathrm{j} \omega)\right)^{T}=\left[\begin{array}{ll}
0.999 & 0.001 \\
0.001 & 0.999
\end{array}\right]
$$

As the off diagonal terms of the RGA matrix are less than 0.2, switching from a SISO controller to a MIMO controller would not have a significant effect on systems performance. 


\section{Chapter 6 - Simulation and Results}

\subsection{Description of Simulator}

The entire wind turbine and pitch control system is simulated using Matlab’s Simulink. The FAST subroutines are connected to Simulink using the S-Function block, which integrates custom Fortran routines containing FAST's equations of motion into the Simulink platform [14]. This setup allows different control modules (including pitch control) to be designed and simulated in Simulink, while also using the complete nonlinear aeroelastic wind turbine model implemented in FAST [14].

As described above, the $5 \mathrm{MW}$ reference wind turbine is the model used in FAST. The collective pitch controller used in the simulation is given in section 4.2, and the individual pitch controller is given in section 5.2. Two different simulations are run: collective pitch control alone and the combined collective and individual control. The block diagrams for the simulations are shown below.

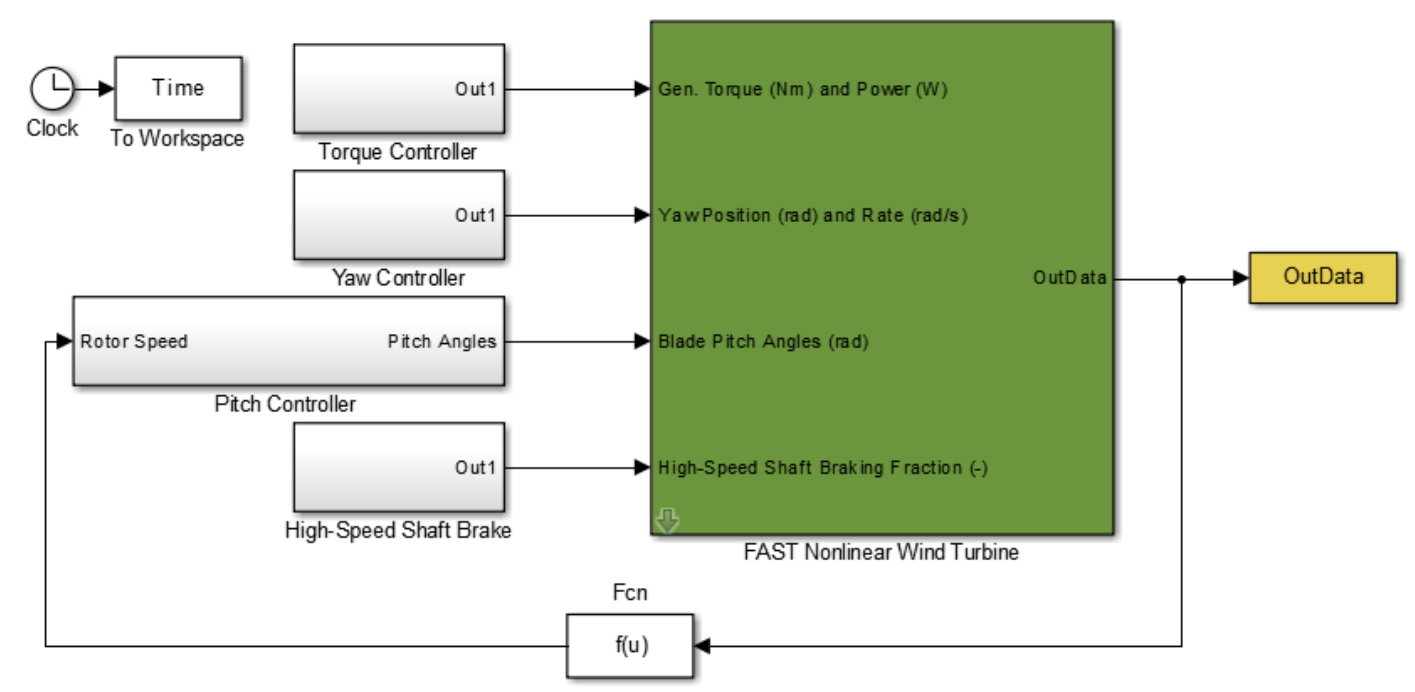

Figure 6.1: Simulation for Collective Pitch Control Only 


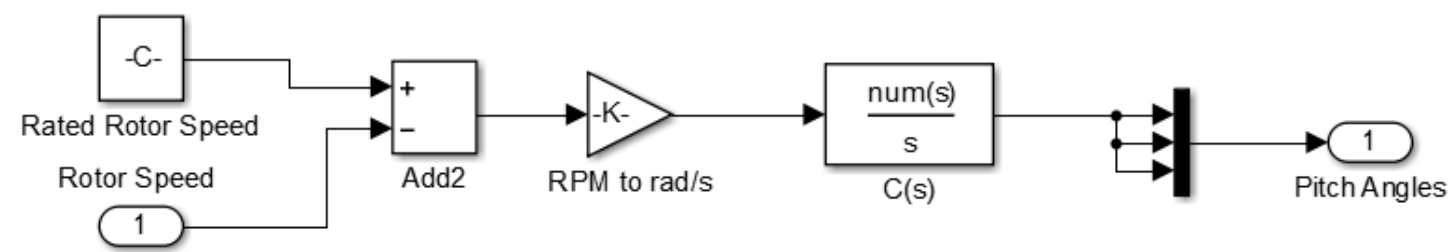

Figure 6.2: Block Diagram of Collective Pitch Controller

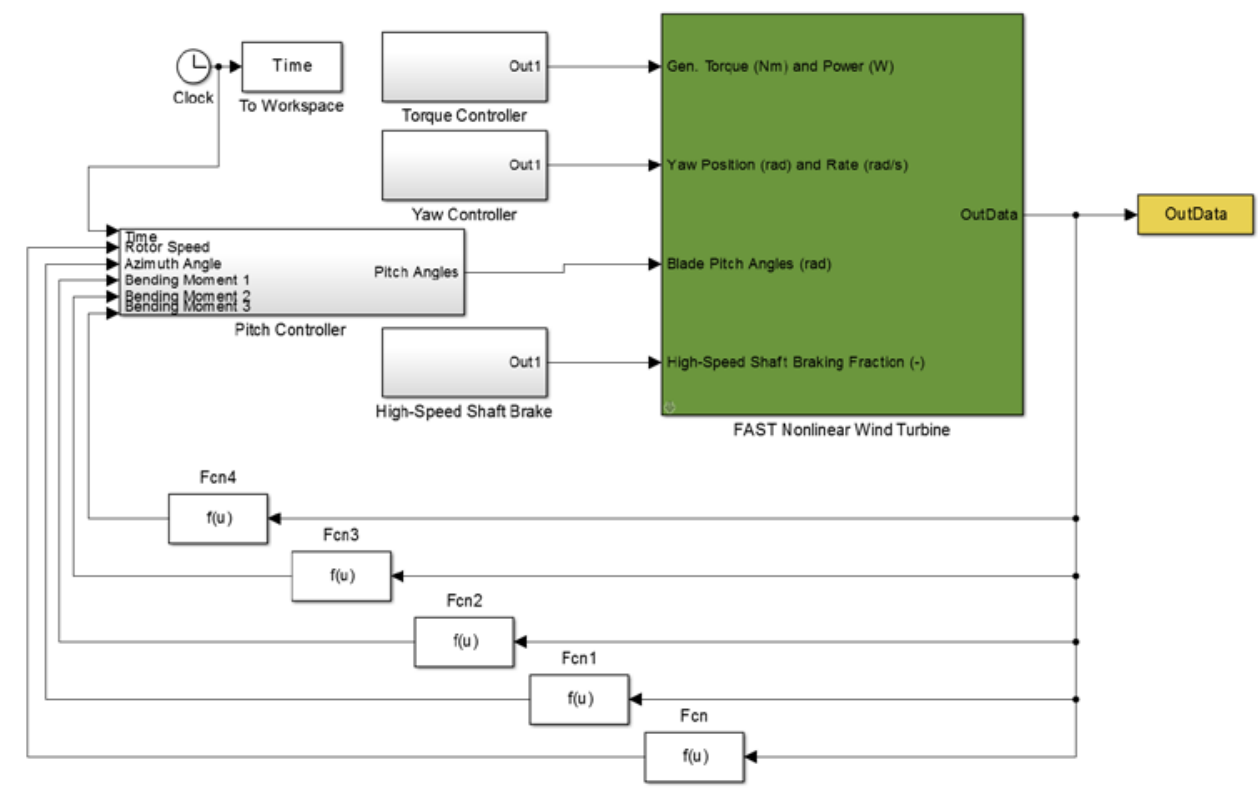

Figure 6.3: Simulation for Collective and Individual Pitch Control

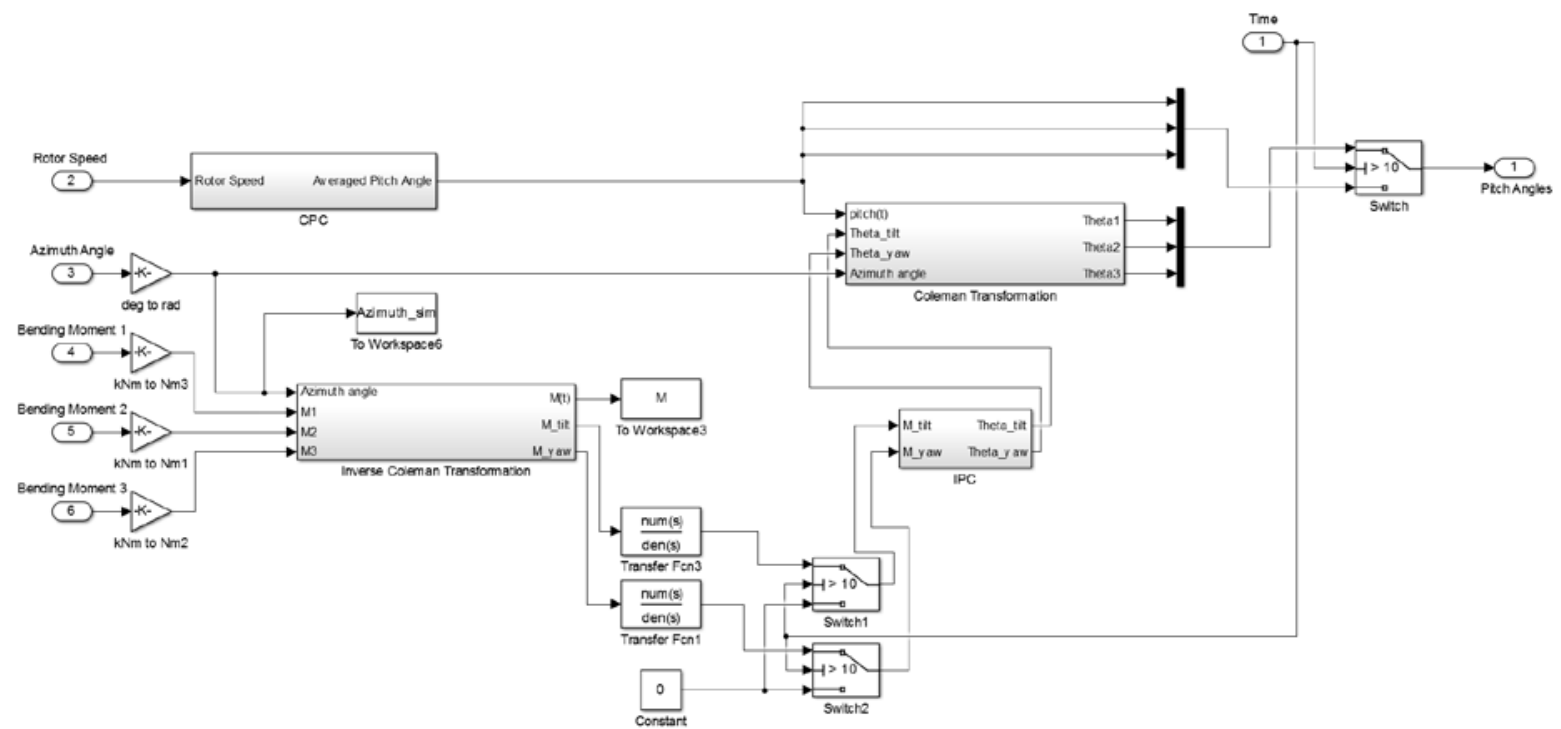

Figure 6.4: Block Diagram of Collective and Individual Pitch Control 
Simple variable speed torque control is used in the simulations along with the pitch controllers designed above. The simulations are run for $300 \mathrm{~s}$ (5 minutes). The input wind is a turbulent wind field with a mean wind speed of $17 \mathrm{~m} / \mathrm{s}$.

\subsection{Results}

The selected results of the simulations are given below. First, the pitch angles of each blade are shown for the duration of the simulation. Then, the pitch angles of each blade are shown for a period of approximately 20 seconds.

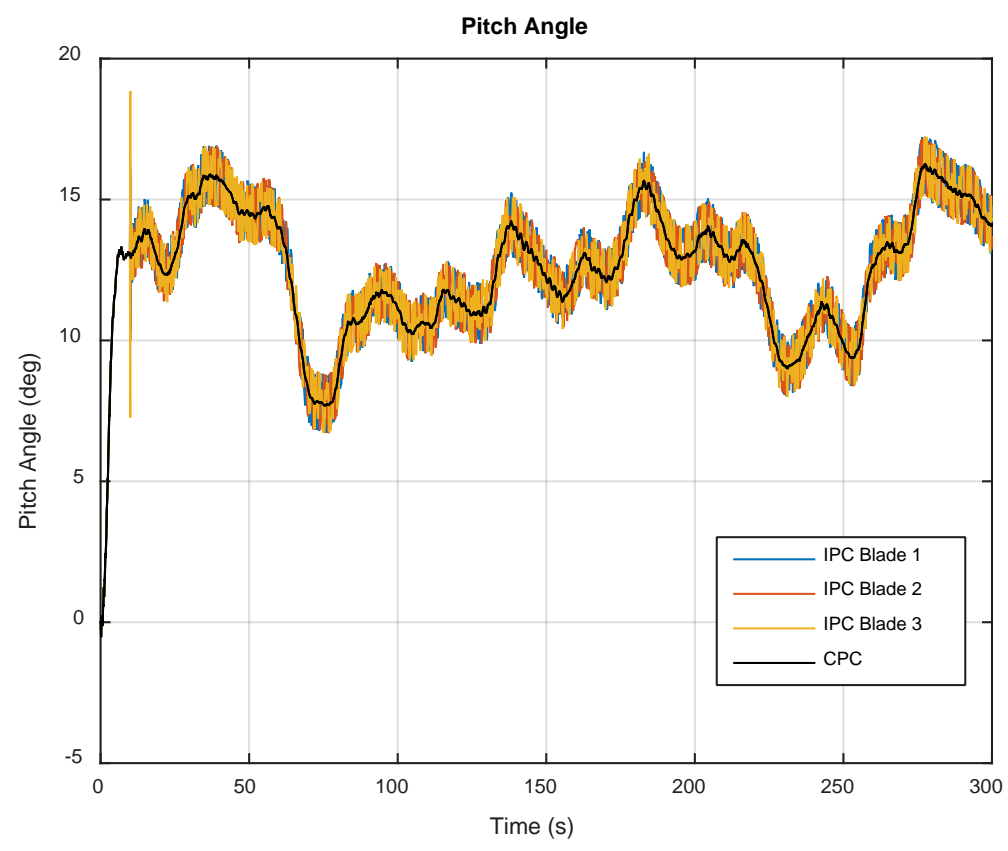

Figure 6.5: Pitch Angles Results from Simulation

It can be seen from Figure 6.6 that, with the individual pitch control, the pitch of each blade oscillates around the pitch angle given by the collective pitch control. The peak of each oscillation occurs at different times for each blade, and the period of oscillation is roughly $1.7 \mathrm{~s}$. This is the inverse of the $3 p$ frequency of $0.6 \mathrm{~Hz}$. 


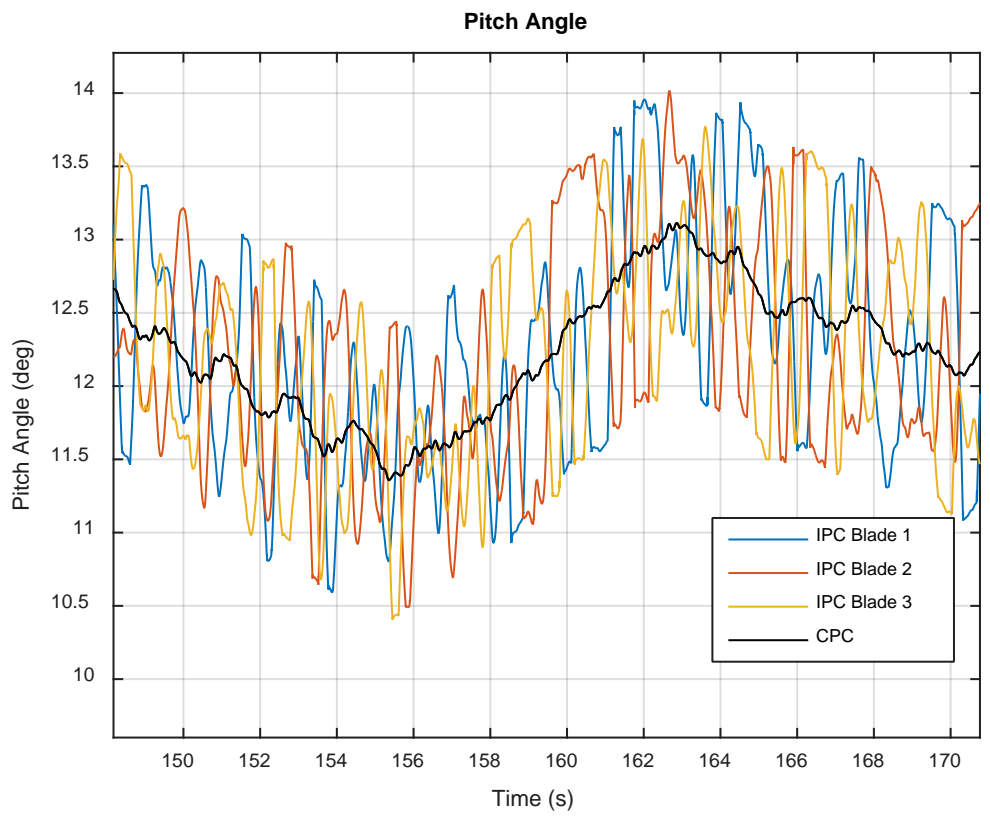

Figure 6.6: Sample of Pitch Angles Results from Simulation

Next, the generator power, generator torque and rotor speed for both the collective pitch control, and the combination of collective and individual pitch control, are shown.

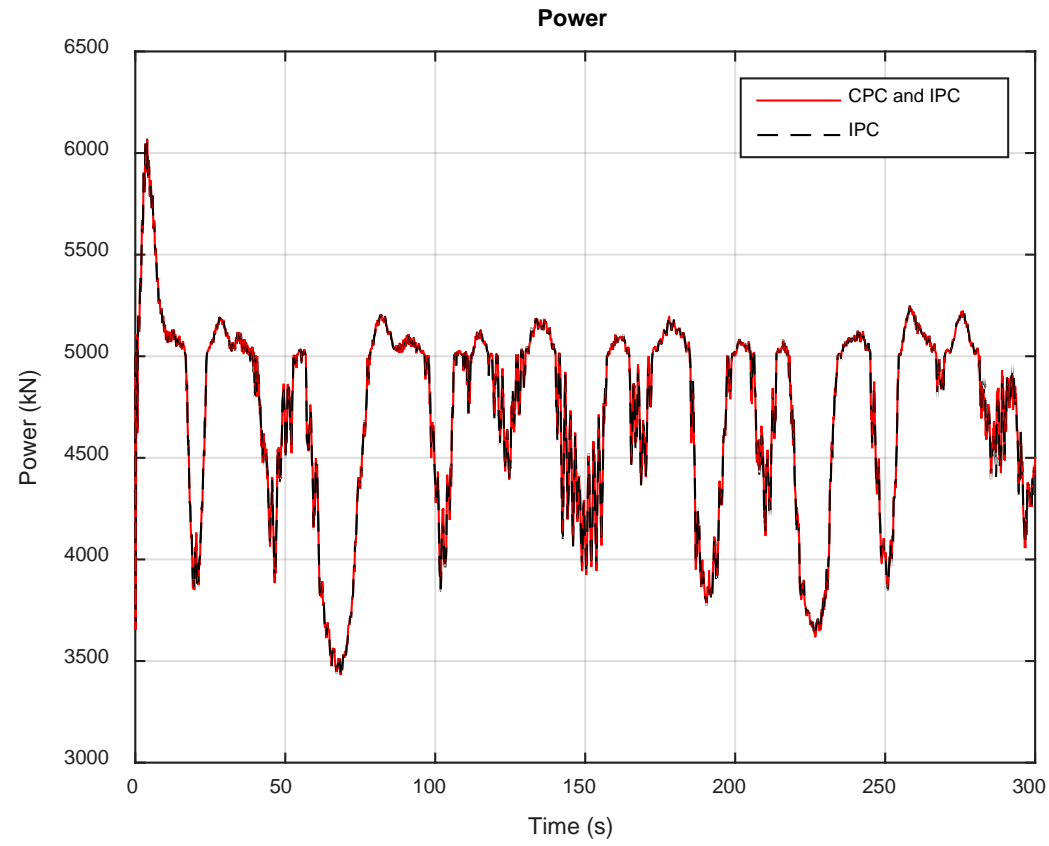

Figure 6.7: Generator Power Simulation Results 


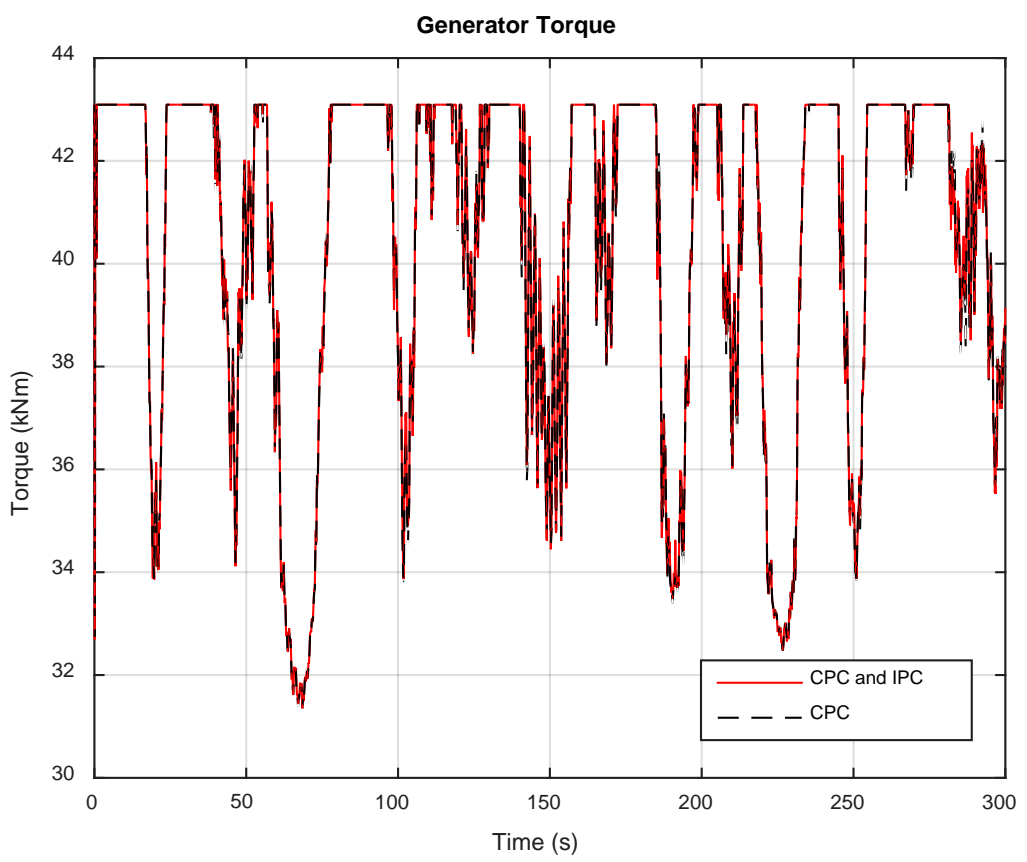

Figure 6.8: Generator Torque Simulation Results

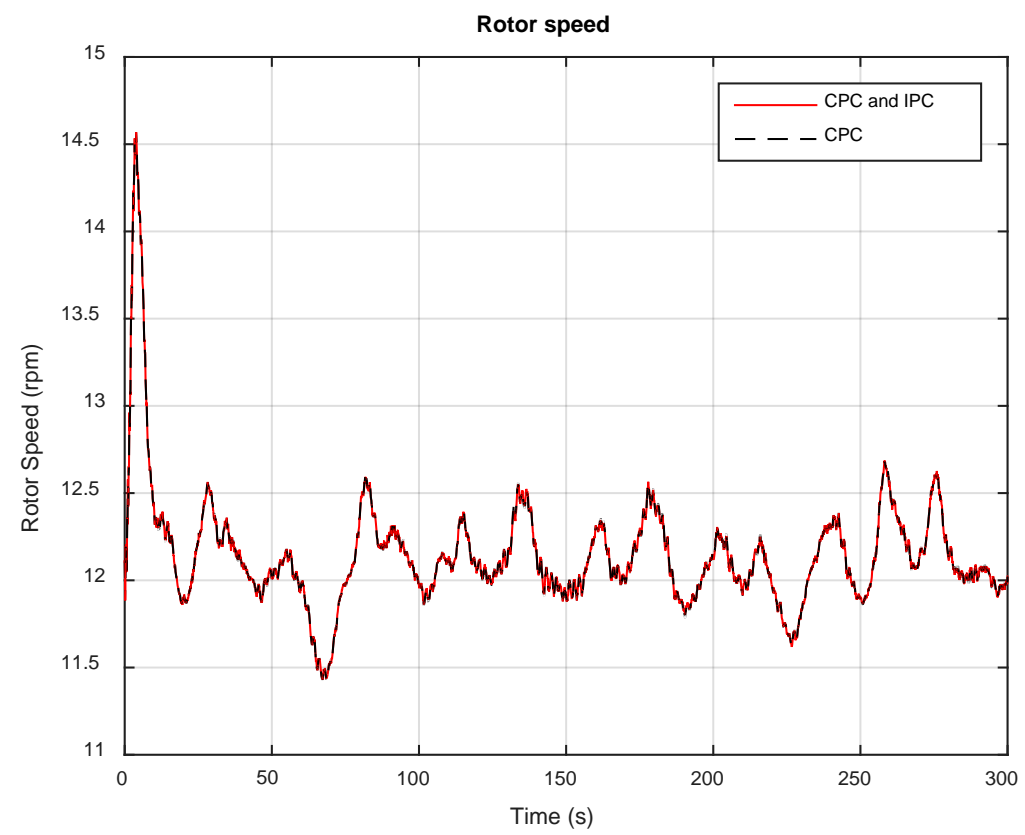

Figure 6.9: Rotor Speed Simulation Results

The plots above show that the generator power, generator torque and rotor speed do not change when the individual pitch control is applied to the simulation. Next, the plots of 
$\mathrm{M}_{\mathrm{tilt}}$ and $\mathrm{M}_{\text {yaw }}$ are shown. Figures 6.10 through 6.13 show that the use of the individual pitch controller reduces both the tilt and yaw bending moments.

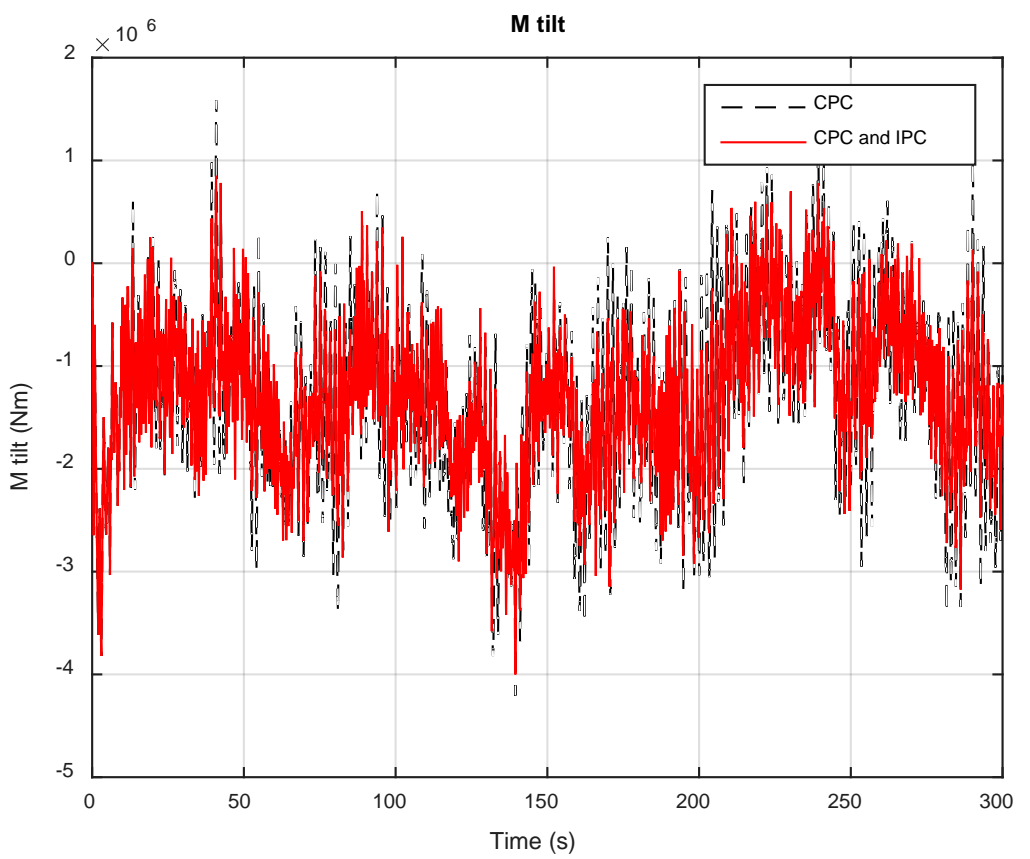

Figure 6.10: $\mathrm{M}_{\text {tilt }}$ Simulation Results

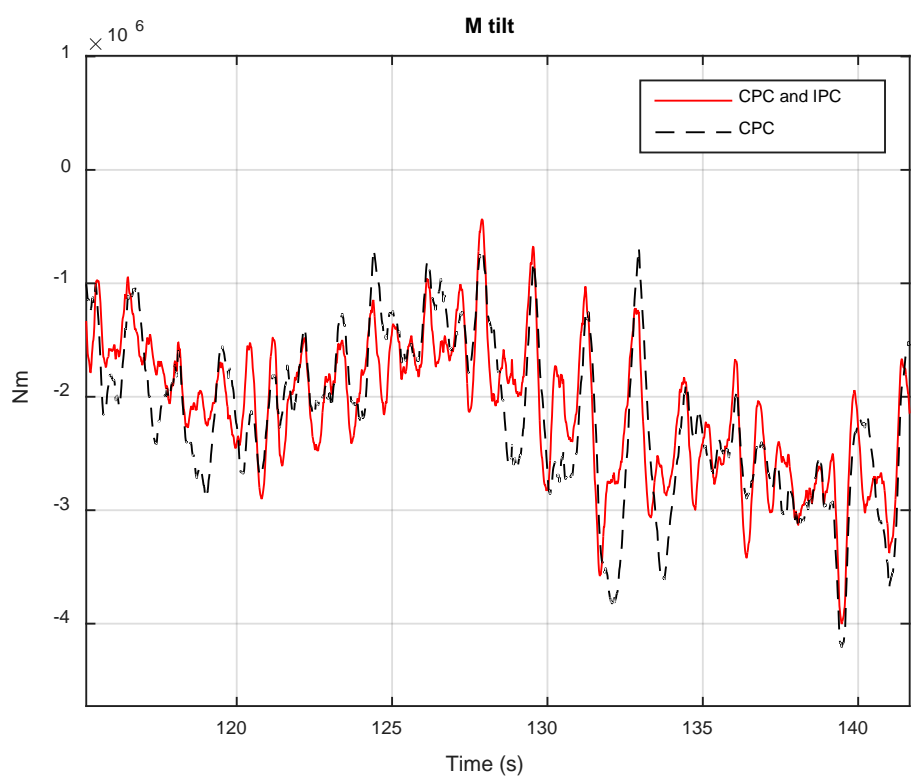

Figure 6.11: Sample of Mtilt Simulation Results 


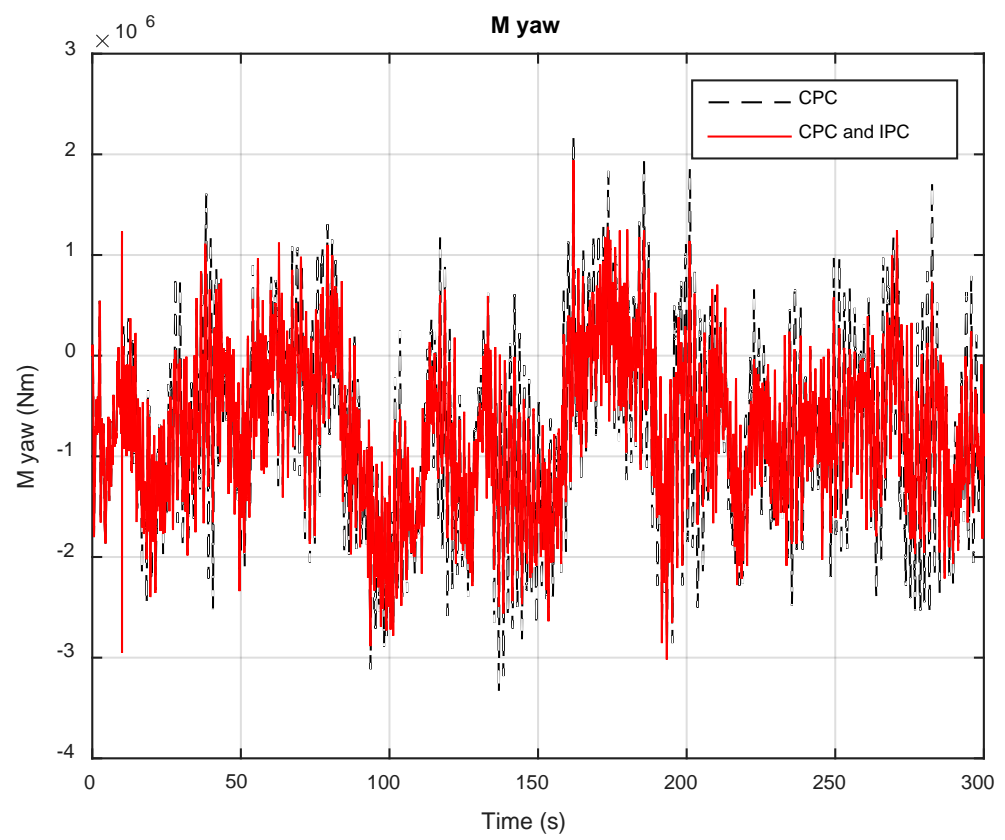

Figure 6.12: $\mathrm{M}_{\text {yaw }}$ Simulation Results

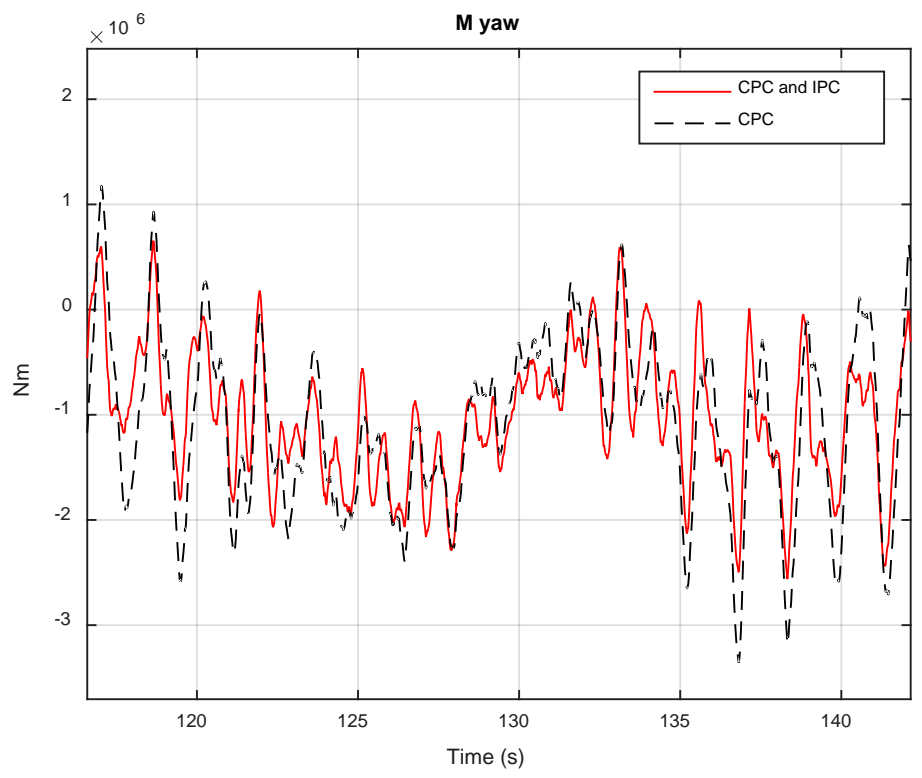

Figure 6.13: Sample of Myaw Simulation Results

The reduction in $\mathrm{M}_{\text {tilt }}$ and $\mathrm{M}_{\text {yaw }}$ can also be seen in the absolute value of the magnitude plots of the Fast Fourier Transforms of the tilt and yaw bending moments, shown in Figures 6.14-6.17. The Fast Fourier Transform is calculated using a sampling 
time of 0.001. Two different selections of data are used for the Fast Fourier Transform. The first selection is the data from 50 to 250 seconds, while the second selection is from 100 to 130 seconds.

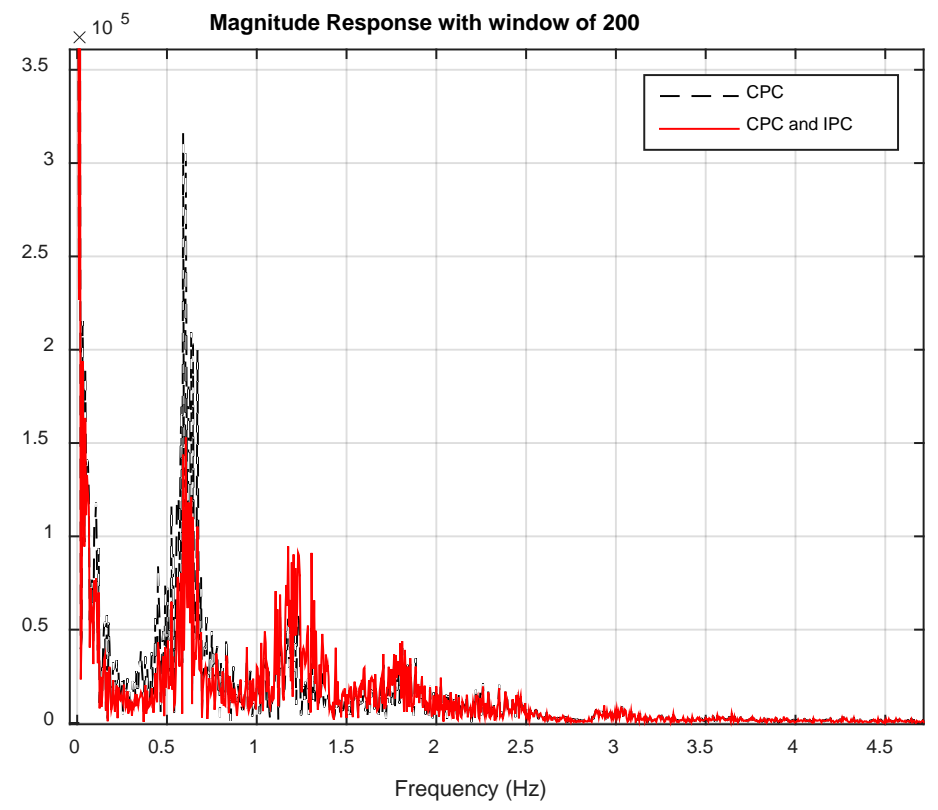

Figure 6.14: Magnitude of Fast Fourier Transform of Mtilt with a window of 200

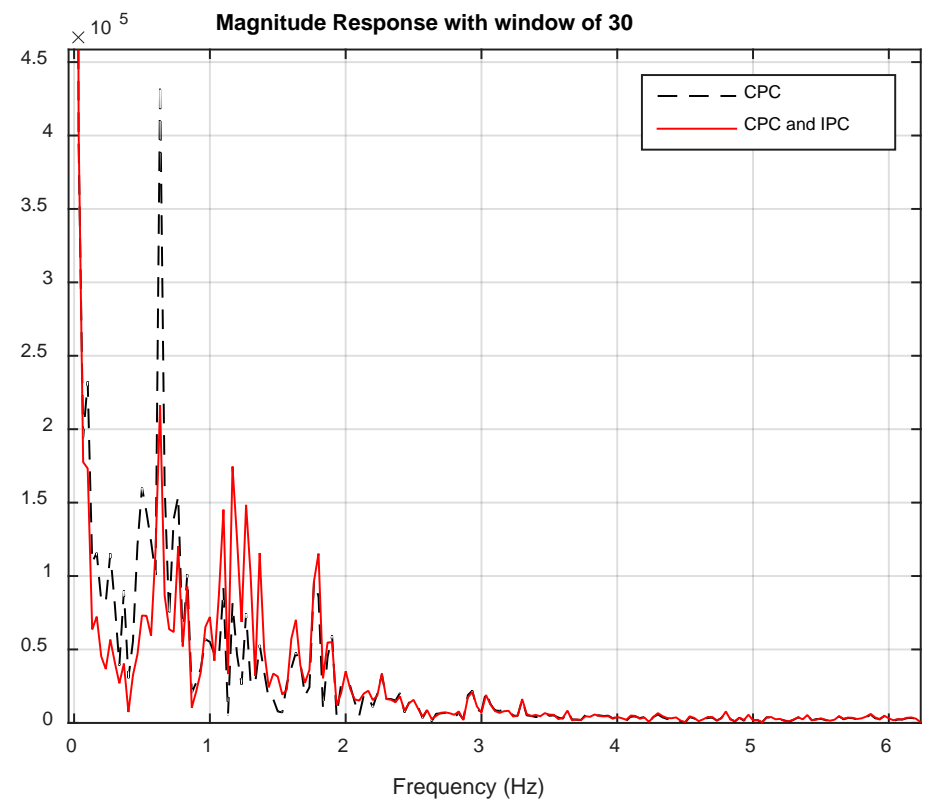

Figure 6.15: Magnitude of Fast Fourier Transform of $\mathrm{M}_{\text {tilt }}$ with a window of 30 
These different selections are used to show changes in loads with the use of the individual pitch controller are not due to the portion of the results selected. The frequencies of interest are $1 p$ and $3 p$ frequencies.

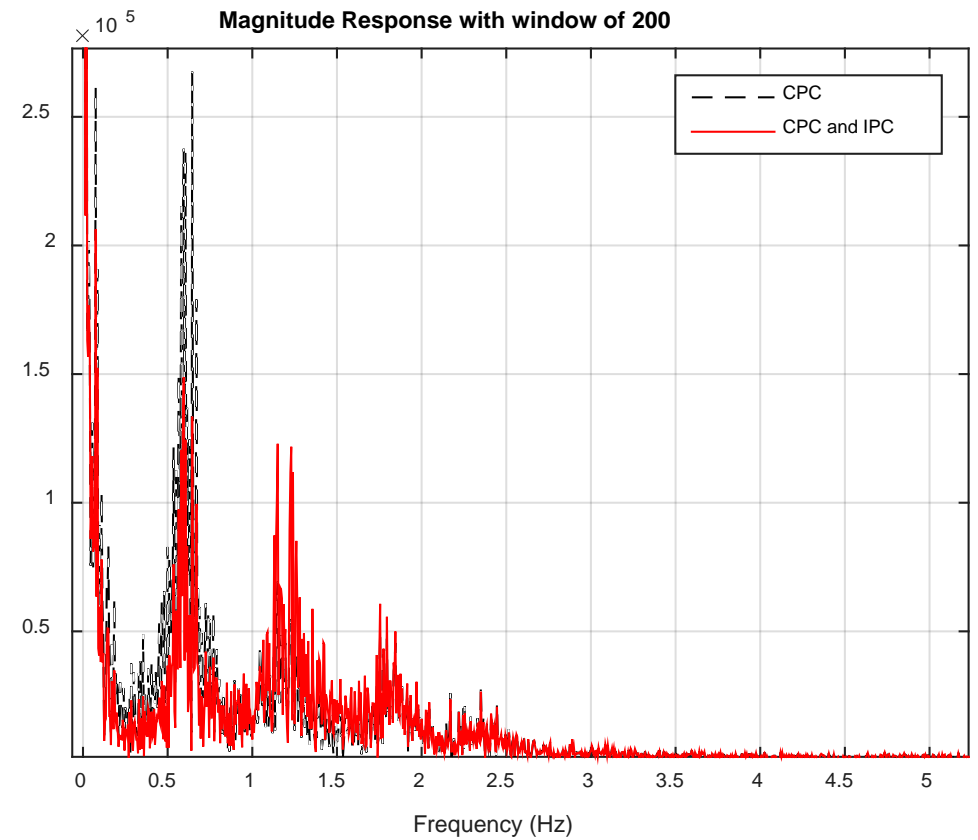

Figure 6.16: Magnitude of Fast Fourier Transform of $M_{\text {yaw }}$ with window of 200

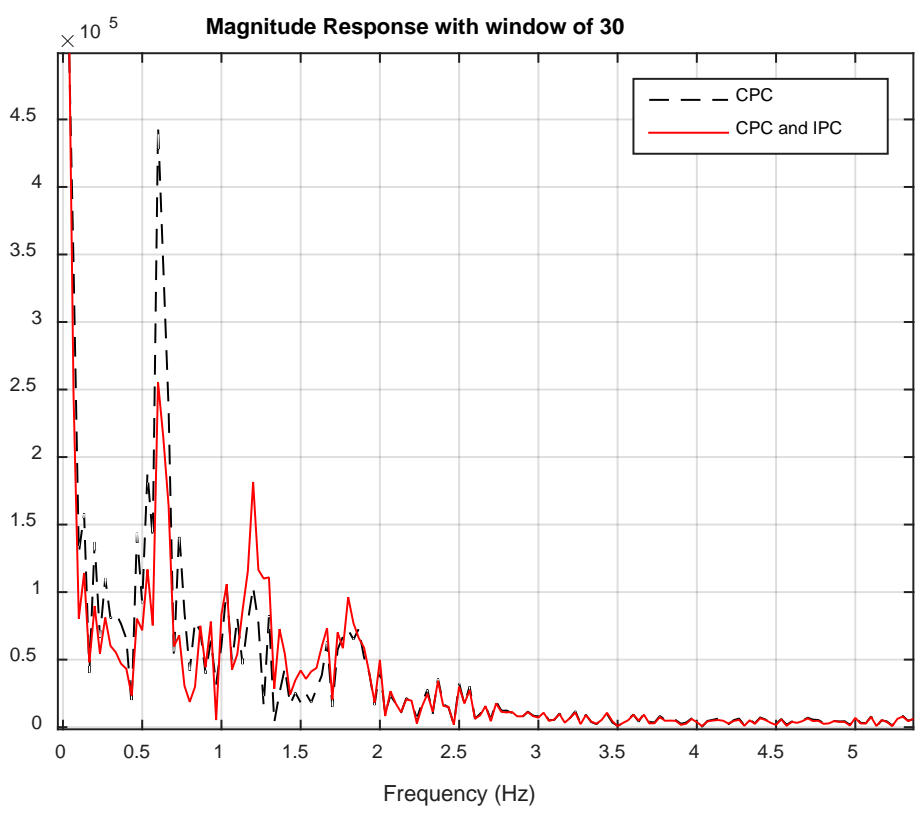

Figure 6.17: Magnitude of Fast Fourier Transform of $\mathrm{M}_{\text {yaw }}$ with window of 30 
The $1 \mathrm{p}$ frequency represents the increase in loads on the tower and nacelle that occur once a rotation due to the imbalances in the rotor. Therefore, the $1 \mathrm{p}$ frequency is equal to the rated rotor speed of $1.27 \mathrm{rad} / \mathrm{s}$, or $0.2 \mathrm{~Hz}$. The 3p frequency of $0.6 \mathrm{~Hz}$ represents the increase in loads on the tower and nacelle that occurs each time a blade is directly in line with the tower. This occurs three times per revolution.

Analysis of the magnitude plot of the Fast Fourier Transform of $\mathrm{M}_{\text {tilt }}$ with a window of 200 seconds shows that the use of the individual pitch controller results in a $28.1 \%$ reduction in bending moment at the $1 p$ frequency and a $20.1 \%$ reduction at the $3 p$ frequency. Similar analysis of the magnitude plot of the Fast Fourier Transform of $\mathrm{M}_{\mathrm{yaw}}$ reveals a $46 \%$ reduction in bending moment at the $1 p$ frequency and a $63.2 \%$ reduction in bending moment at the 3p frequency with the use of the individual pitch controller. When the 30 second window is used, the reductions in the tilt coordinate frame are $45.6 \%$ and $50.3 \%$ at the $1 p$ and $3 p$ respectively. In the yaw coordinate frame, a $34.6 \%$ reduction is found at the $1 p$ frequency while a $42.7 \%$ reduction is shown at the $3 p$ frequency. These results are also presented in Table 6.1.

\begin{tabular}{|l|r|r|}
\hline Window of 200 & M_tilt & M_yaw \\
\hline 1p & $28.9 \%$ & $46.0 \%$ \\
\hline 3p & $20.1 \%$ & $63.2 \%$ \\
\hline Window of 30 & & \\
\hline 1p & $45.6 \%$ & $34.6 \%$ \\
\hline 3p & $50.3 \%$ & $42.7 \%$ \\
\hline
\end{tabular}

Table 6.1: Bending Moment Reduction at Key Frequencies

The magnitude plots of the Fast Fourier Transforms of the root flapwise bending moment of each blade (given in Figures 6.18-6.23) show reductions at the 1p and $2 \mathrm{p}$ frequencies. 


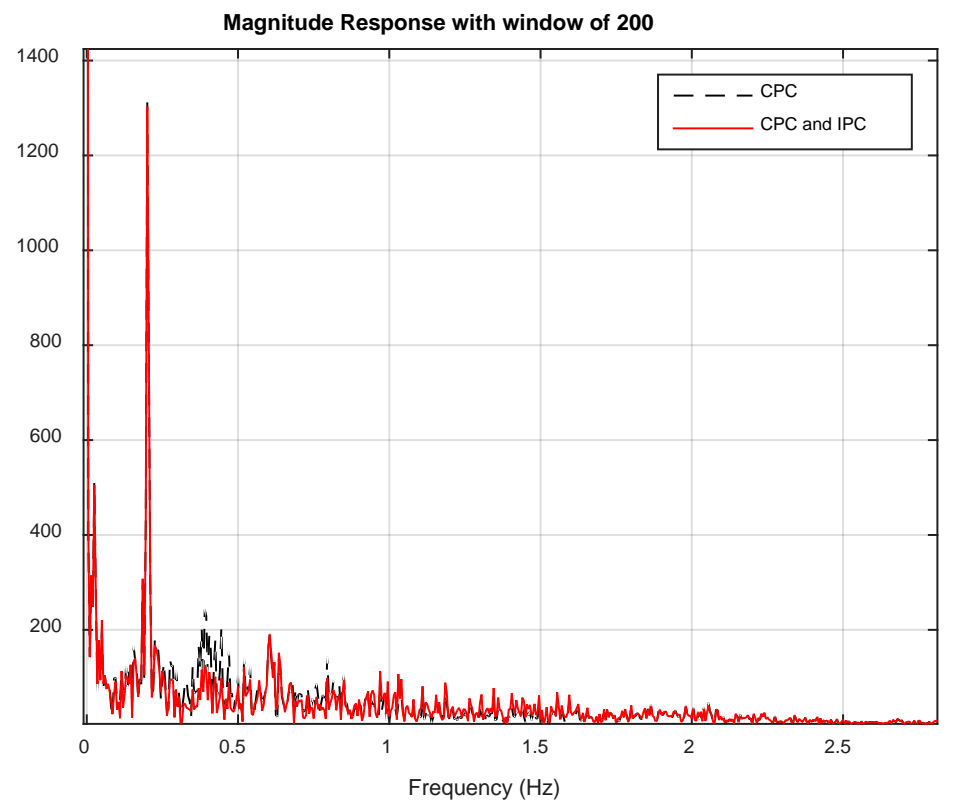

Figure 6.18: Magnitude of Fast Fourier Transform of Bending Moment of Blade 1 with a window of 200

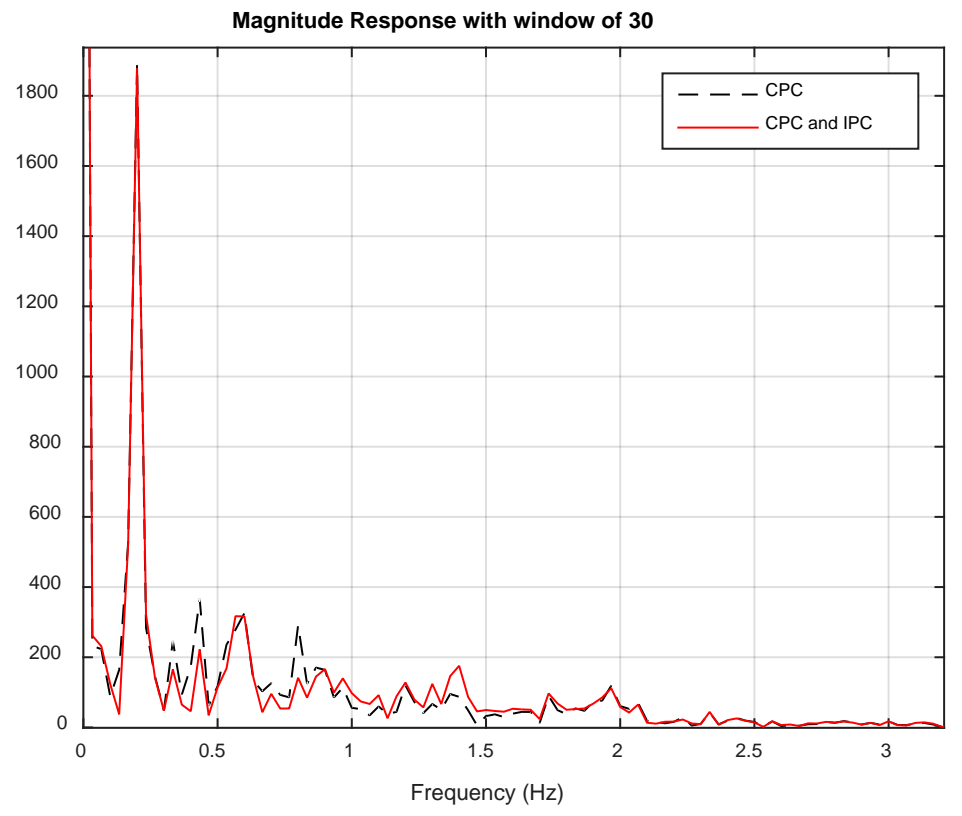

Figure 6.19: Magnitude of Fast Fourier Transform of Bending Moment of Blade 1 with a window of 30 


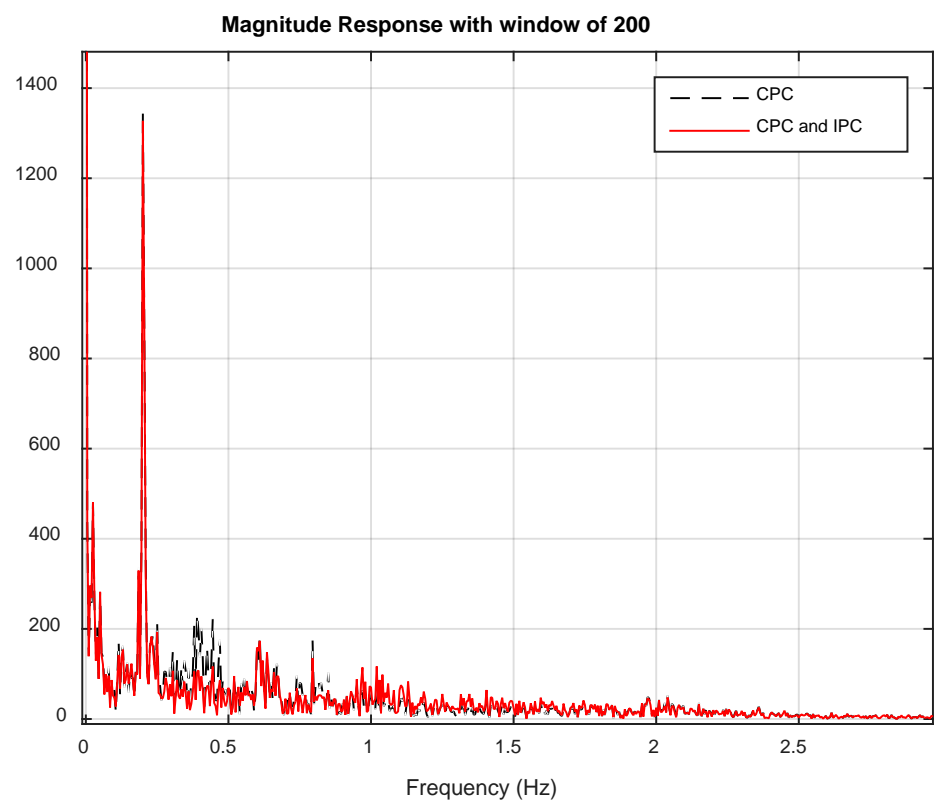

Figure 6.20: Magnitude of Fast Fourier Transform of Bending Moment of Blade 2 with a window of 200

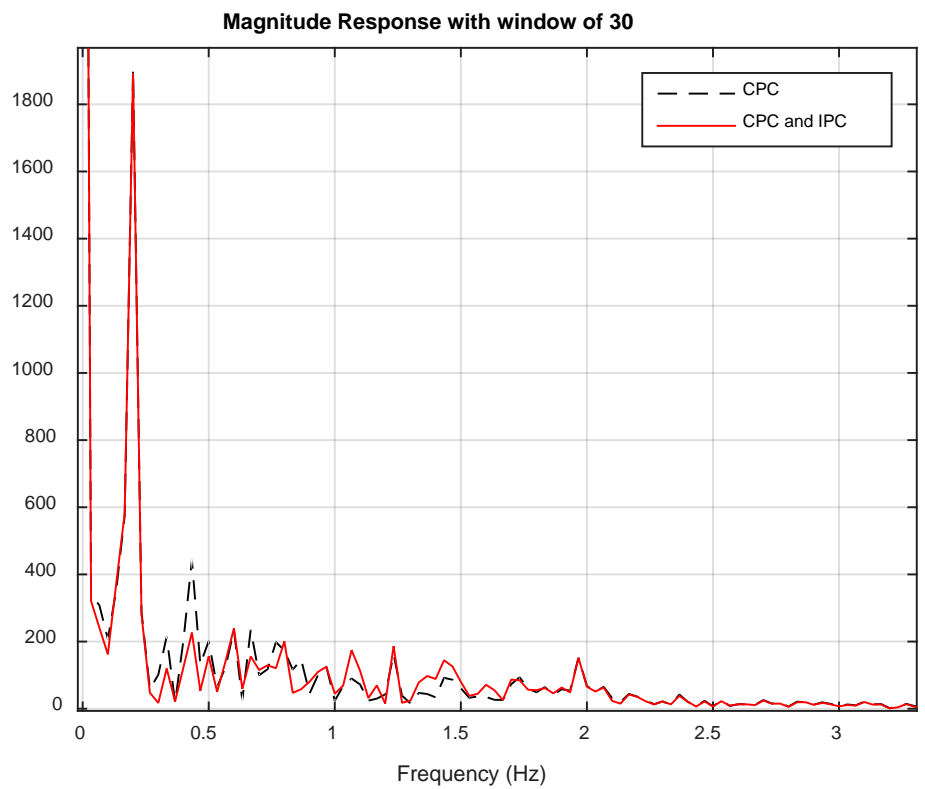

Figure 6.21: Magnitude of Fast Fourier Transform of Bending Moment of Blade 2 with a window of 30 


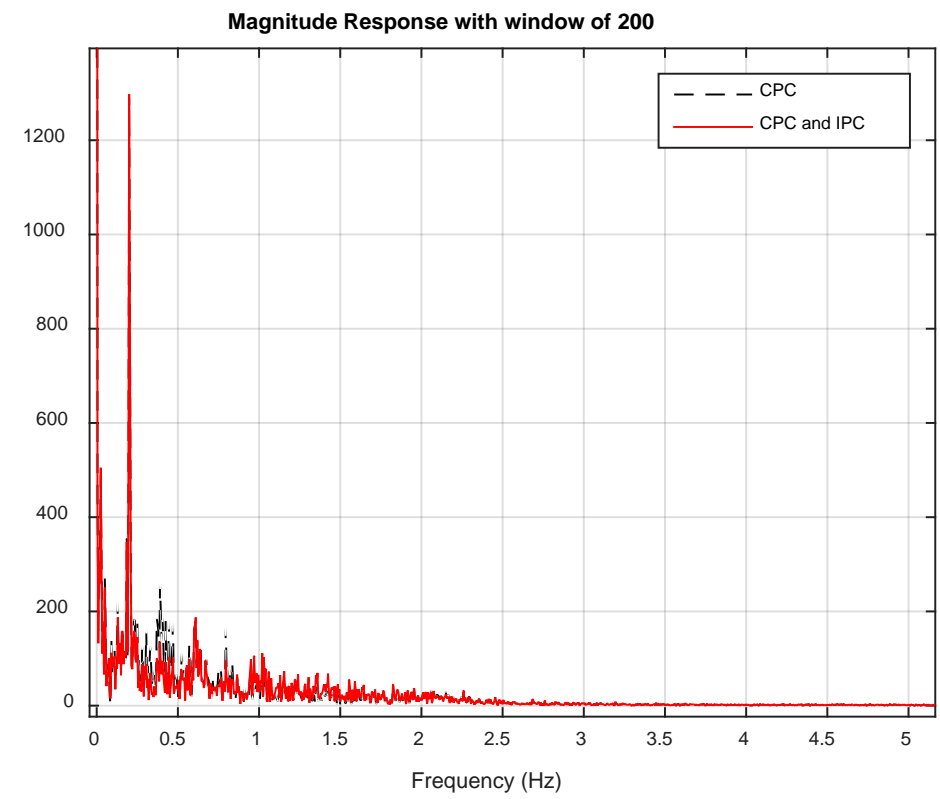

Figure 6.22: Magnitude of Fast Fourier Transform of Bending Moment of Blade 3 with a window of 200

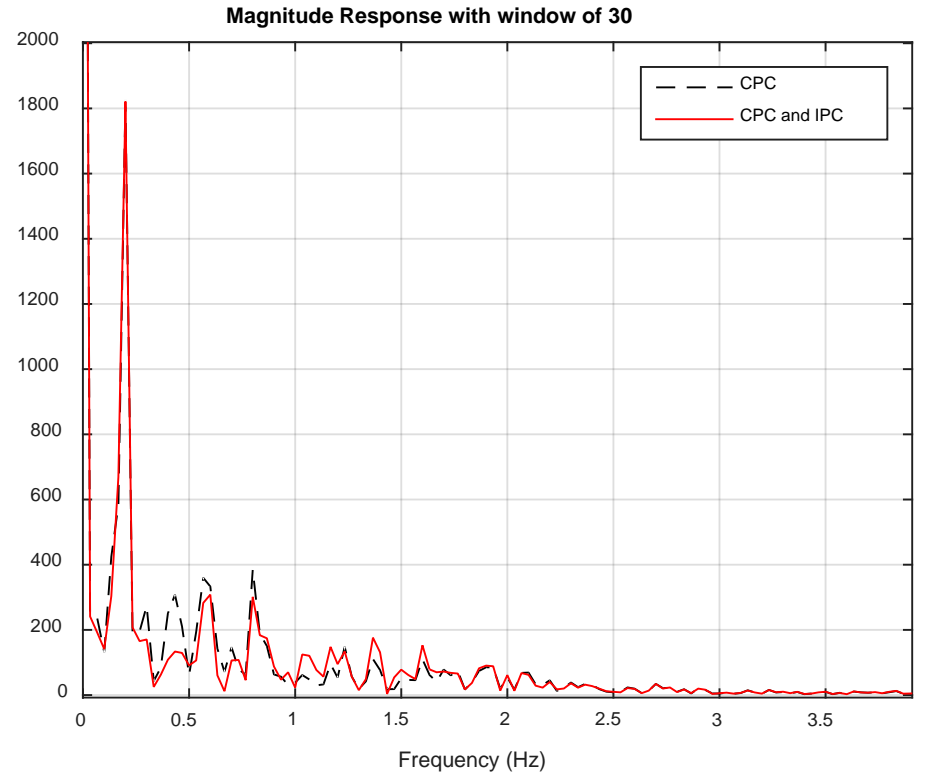

Figure 6.23: Magnitude of Fast Fourier Transform of Bending Moment of Blade 3 with a window of 30 
The $1 \mathrm{p}$ frequency of $0.2 \mathrm{~Hz}$ represents the increased loads on the blade when it passes by the tower, which occurs once per revolution. The $2 \mathrm{p}$ frequency of $0.4 \mathrm{~Hz}$ is the second harmonic of the $1 p$ effect.

The reductions at the $1 \mathrm{p}$ frequency are minimal, at $0.9 \%, 1.6 \%$, and $0.4 \%$ for blades 1, 2, and 3, respectively, with a window of 200 seconds. When a window of 30 seconds is used, the reductions at the $1 \mathrm{p}$ frequency are $0.6 \%, 0.5 \%$ and $0.3 \%$ for blades 1 , 2, and 3. At the $2 \mathrm{p}$ frequency, with a window of 200 seconds, the reductions are larger: $50.7 \%$ for blade $1,40.6 \%$ for blade 2 , and $50.3 \%$ for blade 3 . When a window of 30 seconds is used, the reductions at the $2 p$ frequency are $73.3 \%$, 39.5\% and $56.7 \%$ for blades 1, 2, and 3 respectively. These results are shown in Table 6.2.

\begin{tabular}{|l|r|r|r|}
\hline Window of 200 & \multicolumn{2}{|c|}{ M1 } & \multicolumn{2}{|c|}{ M3 } \\
\hline $1 \mathrm{p}$ & $0.9 \%$ & $1.6 \%$ & $0.4 \%$ \\
\hline $2 \mathrm{p}$ & $50.7 \%$ & $40.6 \%$ & $50.3 \%$ \\
\hline Window of 30 & & & \\
\hline $1 \mathrm{p}$ & $0.6 \%$ & $0.5 \%$ & $0.3 \%$ \\
\hline $2 \mathrm{p}$ & $73.3 \%$ & $39.5 \%$ & $56.7 \%$ \\
\hline
\end{tabular}

Table 6.2: Root Flapwise Bending Moment Reduction at Key Frequencies The variance in percentage reduction at the key frequencies is expected because of the turbulent wind input. The results presented in Tables 6.1 and 6.2 show that the selection of data for the Fast Fourier Transform does not change the order of magnitude of the percentage reduction in bending moment.

Next, additional analysis is performed based on the Fast Fourier Transform. The magnitude response of the Fast Fourier Transform is squared. The plots of the magnitude response of the Fast Fourier Transform squared are similar in shape to the plots of the absolute magnitude response of the Fast Fourier Transform. The percentage reduction of the bending moments at the key frequencies is calculated by examining the area under the 
curve around the frequencies of interest. The results of these calculations are shown in Tables 6.3 and 6.4 below.

\begin{tabular}{|l|r|r|}
\hline Window of 200 & M_tilit & M_yaw \\
\hline $1 p$ & $62.4 \%$ & $71.2 \%$ \\
\hline 3p & $71.8 \%$ & $68.8 \%$ \\
\hline Window of 30 & M_tilit & M_yaw \\
\hline 1p & $70.7 \%$ & $46.4 \%$ \\
\hline 3p & $68.7 \%$ & $61.9 \%$ \\
\hline
\end{tabular}

Table 6.3: Bending Moment Reduction at Key Frequencies using Energy Measurement

\begin{tabular}{|l|r|r|r|}
\hline Window of 200 & M1 & \multicolumn{1}{l|}{ M2 } & M3 \\
\hline $1 \mathrm{p}$ & $0.74 \%$ & $3.20 \%$ & $1.38 \%$ \\
\hline 2p & $71.3 \%$ & $76.6 \%$ & $74.1 \%$ \\
\hline Window of 30 & M1 & M2 & M3 \\
\hline $1 p$ & $0.76 \%$ & $0.90 \%$ & $1.22 \%$ \\
\hline 2p & $68.6 \%$ & $72.6 \%$ & $72.5 \%$ \\
\hline
\end{tabular}

Table 6.4: Root Flapwise Bending Moment Reduction at Key Frequencies using Energy Measurement

The tables above show that, even when using a different kind of analysis based on the Fast Fourier Transform, there are still reduction in bending moment at the key frequencies when the individual pitch control is used. 


\section{Chapter 7 - Conclusions and Future Work}

The results above show that the individual pitch controller reduces the tilt and yaw loads on the non-rotating components of the turbine (the nacelle, tower and foundation) at the $1 \mathrm{p}$ and $3 \mathrm{p}$ frequencies and the blade loads at the $1 \mathrm{p}$ and $2 \mathrm{p}$ frequencies without compromising power generation. Therefore, turbines that utilize individual pitch control will require less maintenance, have a longer lifetime, and be more reliable. This will decrease the costs of operating wind turbines, and consequently the cost of wind energy.

As shown in the results section above, the addition of the individual pitch controller to the collective pitch controller results in reductions in tilt bending moments and yaw bending moments. In terms of the loads on the blades, a small reduction in loads at the $1 p$ frequency is achieved with the combination of collective and individual pitch control, compared to the collective pitch controller alone. At the 2p resonant frequency, the use of the individual pitch controller results in a much larger reduction of loads on the blades. These reductions are shown using different types of anaylsis based on the Fast Fourier Transform. The variation in percentage reduction of the root flapwise bending moment on each of the three blades occurs because of the turbulence in the input wind profile. Additionally, there is an increase in some moments at the $6 \mathrm{p}$ frequency.

Reductions of these moments is left for further investigation.

The performance of the controller above cannot be compared to the results found by Lu et al. and Vali et al. because of the differences in the reference wind turbines used in each study. However, the complexity of the controllers can be compared. The individual pitch controller above is second order, which is much lower order than the 
controllers of at least tenth order designed by Lu et al. and Vali et al. using $\mathrm{H}_{\infty}$ methods. Therefore, the individual pitch controller in this thesis is much simpler to design and implement.

The simulation and validation in FAST verify that the simple individual pitch controller designed in this thesis is an attractive option for application in real world turbines. This is due to the similarities between the $5 \mathrm{MW}$ reference turbine and realworld turbines and the complexity of the simulation in FAST. The next step in this area of research is to develop a model-size wind turbine with individual pitch control capabilities. This would allow testing of individual pitch controllers in a wind tunnel. Testing individual pitch control in a wind tunnel would be the next logical step toward implementation of individual pitch control in life-sized, real-world wind turbines. 


\section{Appendix A: $C_{P}$ Curves}

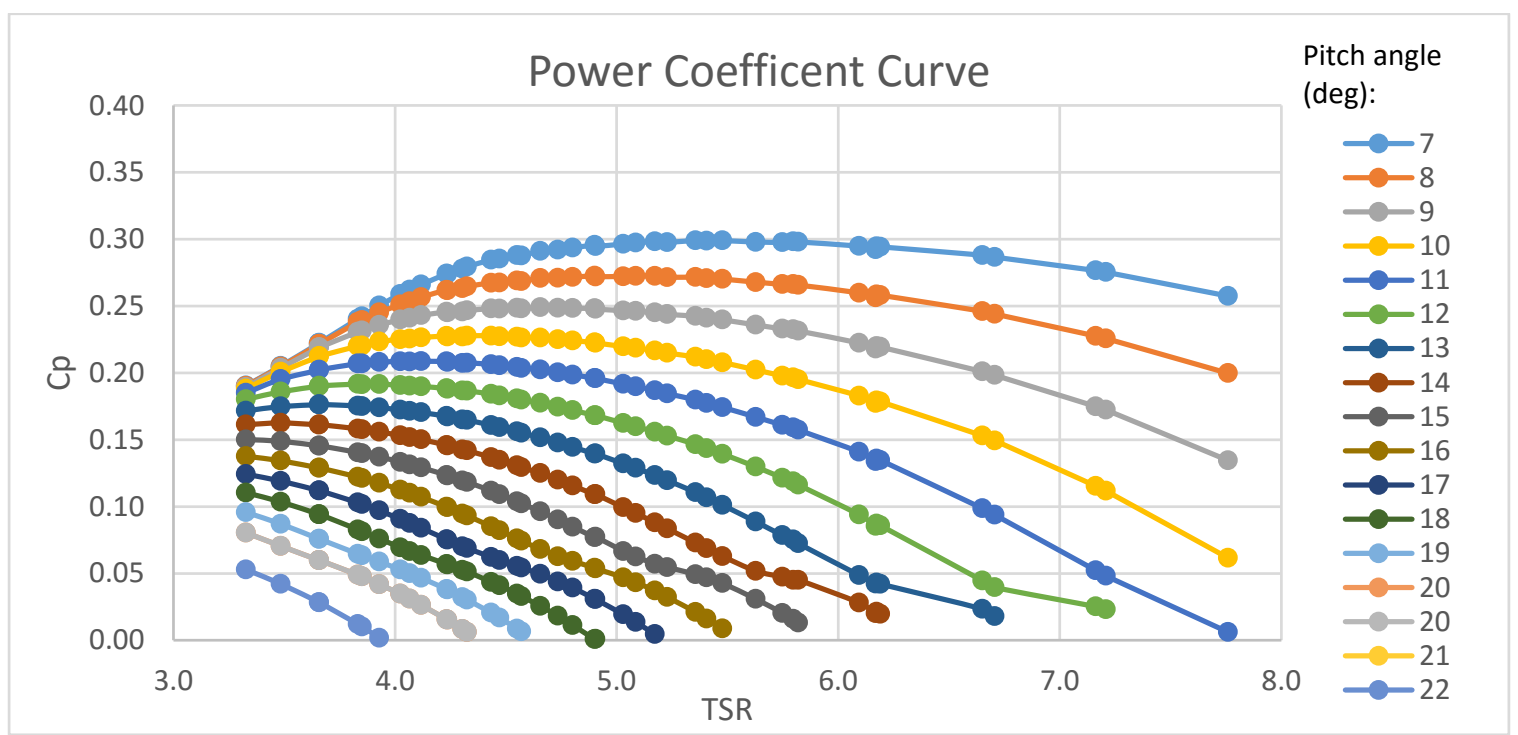

Figure A.1: $\mathrm{Cp} / \lambda$ Curve for the NREL 5 MW Reference Turbine 


\section{Appendix B: Bode Plots of CPC Nominal Plants}

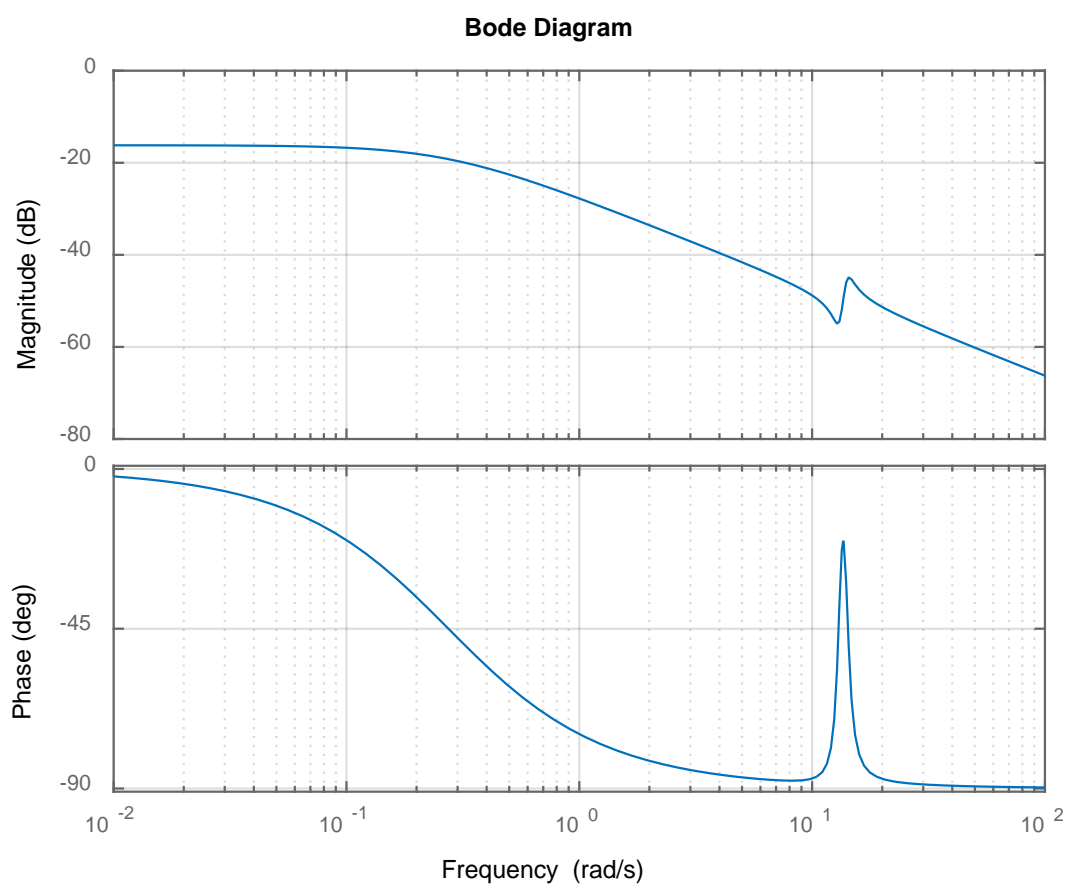

Figure B.1: Bode Plot of Nominal Plant F1(s)
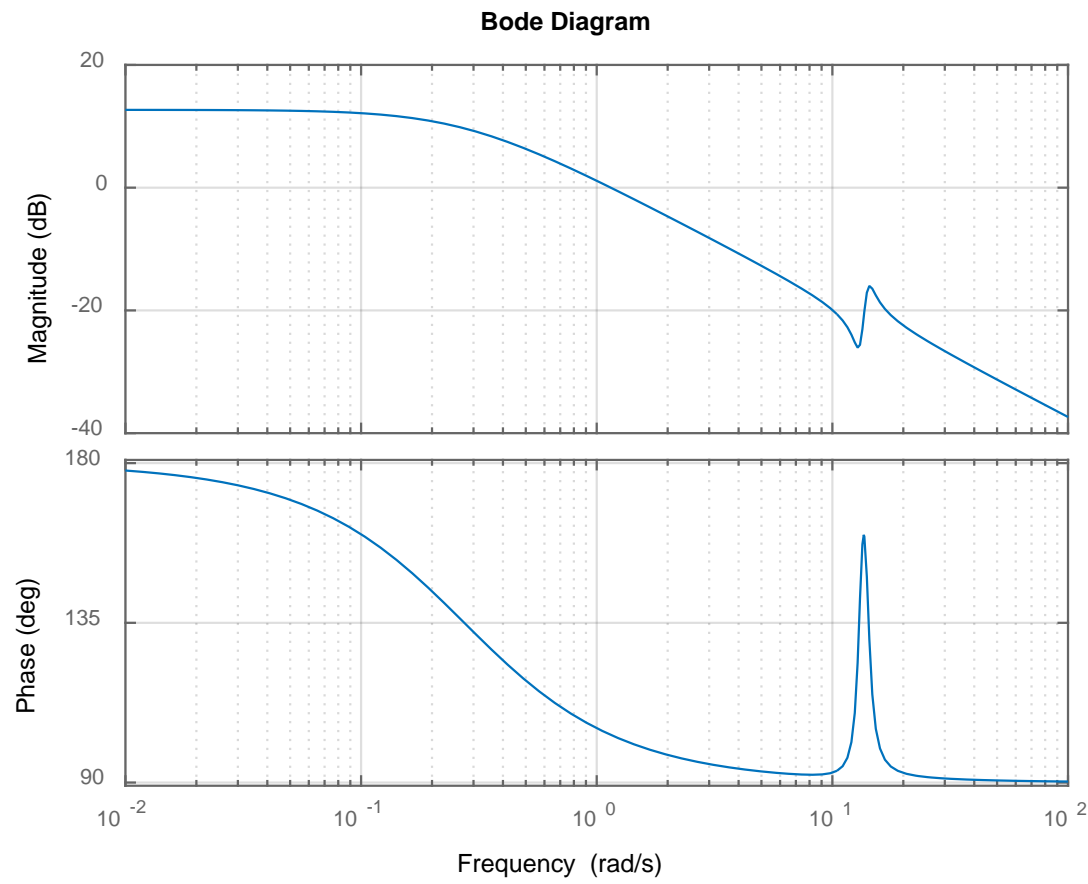

Figure B.2: Bode Plot of Nominal Plant $\mathrm{F}_{2}(\mathrm{~s})$ 

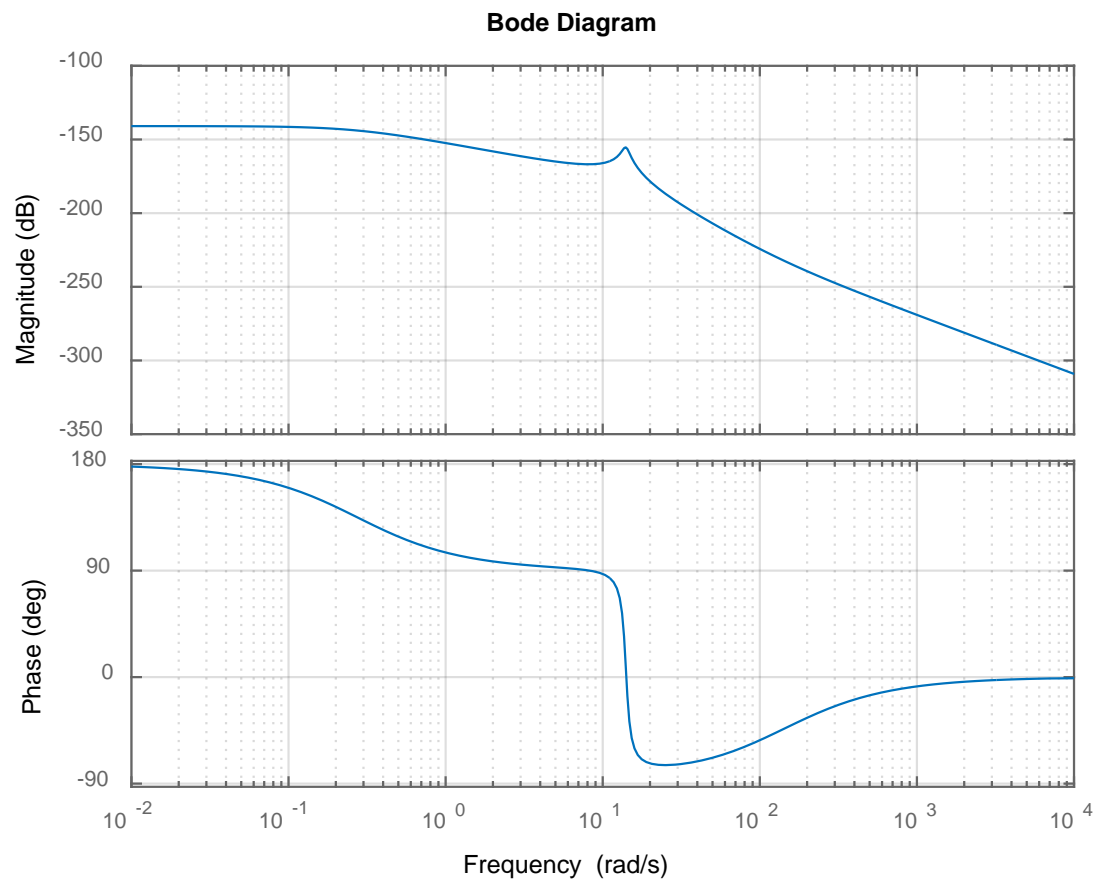

Figure B.3: Bode Plot of Nominal Plant $\mathrm{F}_{3}(\mathrm{~s})$ 


\section{Bibliography}

[1] 10 big wind turbines | Windpower Monthly. (2016). Windpowermonthly.com. Retrieved 11 January 2017, from http://www.windpowermonthly.com/10-biggestturbines

[2] CESC - Control and Energy Systems Center - Case Western Reserve University | QFTCT. (2017). Cesc.case.edu. Retrieved 7 January 2017, from http://cesc.case.edu/OurQFTCT.htm

[3] Chaaban, R. (2012). CpCtCqFiles.zip (1st ed.). Retrieved from $\underline{\text { http://www.rannam.com/downloads/CpCtCqFiles.zip }}$

[4] FAST: blade root flap and edge moments - NWTC. (2017). Wind.nrel.gov. Retrieved 13 January 2017, from https://wind.nrel.gov/forum/wind/viewtopic.php?t=643

[5] Garcia-Sanz, M. (2008-present). Quantitative Feedback Control Toolbox (QFTCT) for Matlab [Computer software].

[6] Garcia-Sanz, M. (2014). Introduction to Wind Energy Systems. Cleveland, OH: Case Western Reserve University.

[7] Garcia-Sanz, M. (2016). Some recommendations to design control specifications. Cleveland, OH: Case Western Reserve University.

[8] Garcia-Sanz, M. \& Houpis, C. (2012). Wind Energy Systems: Control Engineering Design (1st ed.). Boca Raton, FL: CRC Press.

[9] Geyler, M. \& Caselitz, P. (2008). Robust Multivariable Pitch Control Design for Load Reduction on Large Wind Turbines. Journal Of Solar Energy Engineering, 130(3), 031014. http://dx.doi.org/10.1115/1.2931510 
[10] GLOBAL WIND REPORT 2015 | GWEC. (2017). Gwec.net. Retrieved 11 January 2017, from http://www.gwec.net/publications/global-wind-report2/global-wind-report-2015-annual-market-update/

[11] Glower, J. (2002). Nichols Charts (1st ed., p. 3). Fargo, ND: North Dakota State University. Retrieved from http://tinyurl.com/gv4fc4l

[12] Jonkman, J. (2006). NRELOffshrBsline5MW. Golden, Colorado: National Renewable Energy Laboratory. Retrieved from https://www.ieawind.org/AnnexXXIIISecure/Subtask_2S_docs/OC3Files/Baselin eTurbine/NRELOffshrBsline5MW.pdf

[13] Jonkman, J. (2015). FAST | NWTC Information Portal. Nwtc.nrel.gov. Retrieved 10 January 2017, from https://nwtc.nrel.gov/FAST

[14] Jonkman, J. \& Buhl, M. (2005). FAST User’s Guide. Golden, CO: National Renewable Energy Laboratory. Retrieved from http://wind.nrel.gov/public/bjonkman/TestPage/FAST.pdf

[15] Jonkman, J., Butterfield, S., Musial, W. \& Scott, G. (2009). Definition of a 5-MW Reference Wind Turbine for Offshore System Development (NREL/TP-50038060). Golden, Colorado: National Renewable Energy Laboratory. Retrieved from http://www.nrel.gov/docs/fy09osti/38060.pdf

[16] Jonkman, J. \& Jonkman, B. (2016). FAST (Version 8). Golden, CO: National Renewable Energy Laboratory.

[17] Lu, Q., Bowyer, R., \& Jones, B. (2014). Analysis and design of Coleman transform-based individual pitch controllers for wind-turbine load reduction. Wind Energy, 18(8), 1451-1468. http://dx.doi.org/10.1002/we.1769 
[18] Matlab (Version 2015a). (2015). Natick, MA.

[19] Méndez Cubillos, X. \& de Souza, L. (2009). Using of H-Infinity Control Method in Attitude Control System of Rigid-Flexible Satellite. Mathematical Problems In Engineering, 2009, 1-9. http://dx.doi.org/10.1155/2009/173145

[20] Mirzaei, M., Soltani, M., Poulsen, N., \& Niemann, H. (2013). An MPC approach to individual pitch control of wind turbines using uncertain LIDAR measurements. In European Control Conference. Zürich, Switzerland.

[21] Nourdine, S., Camblong, H., Vechiu, I., \& Tapia, G. (2010). Comparison of wind turbine LQG controllers using Individual Pitch Control to alleviate fatigue loads. 18th Mediterranean Conference On Control And Automation, MED'10. http://dx.doi.org/10.1109/med.2010.5547822

[22] NREL 5-MW reference turbine - CP, CQ, CT Coefficients. (2015). National Wind Technology Center Forum. Retrieved 16 January 2017, from https://wind.nrel.gov/forum/wind/viewtopic.php?t=582

[23] Park, S. \& Nam, Y. (2012). Two LQRI based Blade Pitch Controls for Wind Turbines. Energies, 5(12), 1998-2016. http://dx.doi.org/10.3390/en5061998

[24] Schuler, S., Schlipf, D., Cheng, P., \& Allgower, F. (2013). l1-Optimal Control of Large Wind Turbines. IEEE Transactions On Control Systems Technology, 21(4), 1079-1089. http://dx.doi.org/10.1109/tcst.2013.2261068

[25] Sky's the limit? Reducing wind energy costs through increased turbine size. (2016). Utility Dive. Retrieved 11 January 2017, from http://www.utilitydive.com/news/skys-the-limit-reducing-wind-energy-coststhrough-increased-turbine-size/432663/ 
[26] Ungurán, R. \& Kühn, M. (2016). Combined individual pitch and trailing edge flap control for structural load alleviation of wind turbines. In American Control Conference. Boston, MA.

[27] Vali, M., van Wingerden, J., \& Kühn, M. (2016). Optimal Multivariable Individual Pitch Control for Load Reduction of Large Wind Turbines. In American Control Conference. Boston, MA.

[28] Wang, N., Wright, A., \& Johnson, K. (2016). Independent Blade Pitch Controller Design for a Three-Bladed Turbine Using Disturbance Accommodating Control. In American Control Conference. Boston, MA.

[29] Wheeler, L. (2014). Direct Drive Wind Turbine Pitch Control (Undergraduate). Case Western Reserve University. 